\title{
Argiotalus, fils de Smertulitanus, cavalier namnète à Worms (Allemagne) sous Tibère
}

Argiotalus, son of Smertulitanus, a Namnet cavalryman at Worms

(Germany) under Tiberius

Jacques Santrot, Anne Morin et Mathilde Grünewald

\section{OpenEdition}

Journals

Édition électronique

URL : https://journals.openedition.org/rao/635

DOI : $10.4000 /$ rao.635

ISBN : 978-2-7535-1608-3

ISSN : 1775-3732

Éditeur

Presses universitaires de Rennes

Édition imprimée

Date de publication : 20 décembre 2008

Pagination : 187-208

ISBN : 978-2-7535-0789-0

ISSN : 0767-709X

Référence électronique

Jacques Santrot, Anne Morin et Mathilde Grünewald, « Argiotalus, fils de Smertulitanus, cavalier namnète à Worms (Allemagne) sous Tibère », Revue archéologique de l'Ouest [En ligne], 25 | 2008, mis en ligne le 20 décembre 2010, consulté le 23 août 2022. URL : http://journals.openedition.org/rao/635 ; DOl : https://doi.org/10.4000/rao.635 


\title{
Argiotalus, fils de Smertulitanus, cavalier namnète à Worms (Allemagne) sous Tibère
}

\author{
Argiotalus, son of Smertulitanus, a Namnet cavalryman \\ at Worms (Germany) under Tiberius
}

Jacques Santrot*, Anne Morin ${ }^{* *}$ et Mathilde Grünewald***

Résumé : Un moulage de la stèle figurée d'Argiotalus, fils de Smertulitanus, Namnète, cavalier de l'ala Indiana Gallorum, mort à Worms sous Tibère, est entré au musée départemental Dobrée, à Nantes. La relecture du monument funéraire du plus ancien Namnète connu est l'occasion de le situer parmi les stèles de cavaliers auxiliaires de Worms et de Mayence, dans le contexte historique et militaire d'une Gaule en voie de romanisation, mais encore agitée de révoltes comme celle, en 21, des Andes et des Turons, puis du Trévire Iulius Florus et de l'Éduen Iulius Sacrovir.

Summary: A cast from the figurative stela of Argiotalus, son of Smertulitanus, a Namnet cavalryman of the Ala Indiana Gallorum, who died at Worms under Tiberius, has recently been accessed by the Musée départemental Dobrée in Nantes. A re-reading of this oldest known funerary monument of a Namnet provides the opportunity to place it among the stelae of foreign cavalrymen from Worms and Mainz, in the historical and military context of a Gaul in course of Romanization but still troubled by revolts such as, in 21, that of Andi and Turoni, then of the Trevir Iulius Florus and of the Eduan Iulius Sacrovir.

Mots clés : stèle figurée, inscription, Namnètes, Nantes, Argiotalus, Sacrovir, Florus, cavalier, auxiliaires, aile, ala Indiana, Trévires, Worms.

Key words: figurative stela, inscription, Namneti, Nantes, Argiotalus, Sacrovir, Florus, cavalryman, auxiliaries, wing, ala indiana, Treviri, Worms.

En 1666, la stèle d'Argiotalus, fils de Smertulitanus, cavalier namnète enrôlé dans l'armée romaine, est trouvée à Worms, en Rhénanie-Palatinat (Allemagne) ${ }^{1}$. Un moulage de ce monument est désormais conservé au musée départemental Dobrée, à Nantes, et c'est l'occasion de rappeler ce que ce monument apporte à notre connaissance.

La stèle funéraire d'Argiotalus a été trouvée dans la nécropole nord de la ville romaine des Vangions, devant la porte SaintMartin (Martinspforte ou Stadttor St. Martin) lors de la réno- vation des fortifications médiévales, dans une zone qui n'a pas fait l'objet de véritables fouilles archéologiques et qui n'est plus exploitable aujourd'hui (Boppert 1998, p. 21-22). Trois autres monuments funéraires ont été trouvés dans la même nécropole en 1666 : la stèle de Veiagenus, un Rhète de la natio Montanus (Rhétie ou Norique), soldat dans la cohorte des Rhètes et des Vindéliciens (CIL, XIII, 6240; Boppert 1998, nº 58), celle de Q. Carminius Ingenuus, citoyen romain et signifer de l'aile des Espagnols² (CIL XIII, 6233; Esp. 6014; Boppert 1998,

* Conservateur en chef du Patrimoine, directeur du Musée départemental Dobrée (Conseil général de Loire-Atlantique), Nantes. (jsantrot@cg44.fr)

** Docteur en Histoire, enseignante, La Baule (Loire-Atlantique). (christophe.morin871@orange.fr)

*** Directrice du Museum der Stadt Worms im Andreastift, Worms (Allemagne).(museum@worms.de)

1. Worms, Museum der Stadt Worms im Andreasstift, inv. R 1658 : CIL XIII, 6230; Dessau H., Inscriptiones Latinae Selectae, Berlin, 1916 (3e éd., 1962-1968), 2496; Esp. VIII, 6011; Boppert 1998, p. 80-81, n 47, pl. 50; Santrot 2008.

2. Parmi les ailes des Espagnols connues, seule l'ala I Hispanorum est recensée dans le commandement militaire de Mogontiacum-Mayence entre 13 av. J.-C. et 68 apr. J.-C. : Holder 1980, n 493; Selzer 1988, p. 71; Junkelmann 1991, p. 138-139, nº 79. 
$\mathrm{n}^{\circ}$ 49), et celle de l'Helvète Licinus, cavalier de cette même aile (CIL, XIII, 6234; Esp. 6016; Boppert 1998, n 48). La stèle du Trévire Partus, cavalier de l'aile Agrippiana, a été trouvée dans la même zone mais quatre ans plus tard, en 1670 (CIL, XIII, 6235; Boppert 1998, $\mathrm{n}^{\circ}$ 50). Les stèles de Veiagenus et de Partus ont été détruites dès 1689.

La stèle d'Argiotalus est mentionnée pour la première fois en 1671 par J. Tollius dans In adnotatione ad Ausonium (manuscrit, Amsterdam, p. 791) et sa première édition figure dans les Acta Academicae Theodoro-Palatinae (I, Mannheim, 1766, p. 26). En France, elle est connue dès 1899 par le CIL XIII, I, 1, p. 483, en 1905 par le CIL XIII, II, 1, 6230, puis en 1916 (ILS, 2496). Sa photographie est publiée en 1922 dans le Recueil d'Espérandieu (VIII, 6011), puis par Walburg Boppert en 1992 et en 1998 (avec bibliographie détaillée p. 80). La première évocation de cette inscription dans l'Ouest est due au chanoine Georges Durville, bientôt conservateur du musée départemental d'Archéologie à Nantes, qui la publie dans un long article de L'Écho de la Loire, le 17 septembre 1922 : «Le plus ancien soldat Nantais inhumé chez les Boches au $\mathrm{I}^{\mathrm{er}}$ siècle " (sic!). Nous sommes dans le contexte qui succède au traumatisme de la Grande Guerre et l'archéologue nantais, trop âgé pour avoir été mobilisé, ne cite pas le CIL, récemment paru (mais à Berlin!); il renvoie à l'édition de la Notitia Dignitatum et Administrationum par Edouard Bocking (Bonn) et à sa bibliographie de la stèle (1839, I, p. 828 et 1197). Ce n'est qu'en 1927 que Durville publie une photographie de la stèle en frontispice de son Catalogue du Musée archéologique de Nantes, $2^{2}$ partie, Musée lapidaire : ce cliché avait été obtenu dès 1922 par un capitaine dans l'armée d'occupation, le fils de Pitre de Lisle du Dreneuc, second conservateur du musée départemental d'Archéologie de Nantes et prédécesseur de Durville à la tête du musée Thomas Dobrée (Durville 1927, p. 52-57). La photo est ainsi légendée : "Stèle d'un soldat nantais mort sur les bords

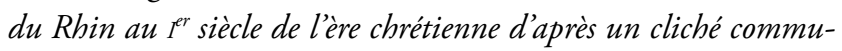
niqué par M. le Directeur de la Bibliothèque de Worms", avec ce commentaire : Argiotalus "était en garnison dans la Germanie, sur les bords du Rhin, au poste d'honneur, contre l'envahissement des barbares d'Outre-Rhin ». (re-sic!). Durville situe la mort du cavalier namnète "probablement au temps de l'empereur Trajan" (p. 52). "L'armée romaine avait une aile dite "l'aile Indienne", autrefois sur les bords du Rhin. Cette aile y cantonnait, d'après les indications fournies par d'autres épitaphes, sous le règne de Trajan» (p. 53).

\section{LE MONUMENT}

Le monument original a été taillé dans un calcaire coquillier gris beige à reflets roux, rubéfié par incendie à hauteur du champ épigraphique. Cette pierre provient de la région de
Mayence et a été apportée à Worms par le Rhin tout proche. De belle apparence, cette stèle reste en assez bon état de conservation bien que brisée en cinq morceaux, recollée au ciment gris avec deux puissantes agrafes de fer scellées sur les côtés (restauration ancienne). Une fracture presque horizontale coupe l'inscription au niveau de la ligne 4 tandis qu'une seconde longe en diagonale l'arrière-main et le dos du cheval en traversant le cavalier, puis se divise vers les bords, près des oreilles, des yeux et de la bouche du cheval. Les bords de la stèle et de sa niche sont épaufrés. Le pied droit et les mains du cavalier ont disparu. La partie haute de la javeline et son talon ont été brisés et perdus; sa pointe déborde sur le rampant gauche du fronton. Le relief est très usé par une longue exposition aux intempéries et tavelé d'une croûte de pollution noirâtre, pourtant allégée par le "dépoussiérage » de 1980. Le dos de la stèle est grossièrement épannelé et légèrement cintré. Toujours exposé à l'air libre, le monument est désormais à l'abri du cloître de l'ancien chapitre (fig. 1 et 2).

Le monument est complet de ses éléments informatifs mais, son pied ayant été brisé horizontalement sous la moulure basse du champ épigraphique, on ne peut plus vérifier si la stèle avait été scellée sur un socle à loculus. Rectangulaire, verticalement allongée, elle est encore haute de $1,36 \mathrm{~m}$, large de $0,59 \mathrm{~m}$ et épaisse de $0,36 \mathrm{~m}$. Son couronnement presque triangulaire imite les rampants d'un fronton, arrondis selon le périmètre de la conque inscrite, avec trois acrotères grossièrement sculptés de palmettes simplifiées, dégénérées. Elle est divisée en deux registres. Au-dessus du champ épigraphique, le défunt figure en " portrait » équestre dans une niche en cul-de-four à godrons gravés en coquille, inscrite dans les rampants du pseudo-fronton. Il est montré dans l'exercice de ses fonctions : cavalier, il charge l'ennemi sur un cheval cabré à droite, dont la queue tendue, "touffue et longue " (Xénophon, cité par Vigneron 1968, p. 8, n. 1 et 2), prolonge l'oblique du corps traité en un volume quasi cylindrique. Malgré l'usure, on reconnaît différentes courroies du harnachement, un anneau, la selle et sa chabraque ornée. Protégé, à gauche, d'un long bouclier ovale de tradition gauloise, le cavalier brandit une javeline du bras droit lancé en arrière. La maladresse de son geste est accrue par l'érosion qui abolit la position du visage et le sens du regard.

D'une mise en page très classique, l'inscription est incisée en capitales sur huit lignes de hauteur décroissante, dans un champ en creux à double encadrement à doucine (sauf sur la bordure inférieure), haute de $61 \mathrm{~cm}$ et large de 43 . La hauteur des lettres est de $6 \mathrm{~cm}$ (1. 1 et 2), $5,5 \mathrm{~cm}$ (1. 3, 4 et 5), $5 \mathrm{~cm}$ (1. 6 et 7), et $4,5 \mathrm{~cm}$ (1. 8). Des ponctuations triangulaires, pointe en bas, séparent les mots (hedera douteuse lue par Boppert, 1. 1) et terminent quatre des huit lignes. Le texte est aligné à gauche et le lapicide a calibré l'espacement des lettres pour utiliser tout le champ épigraphique. 


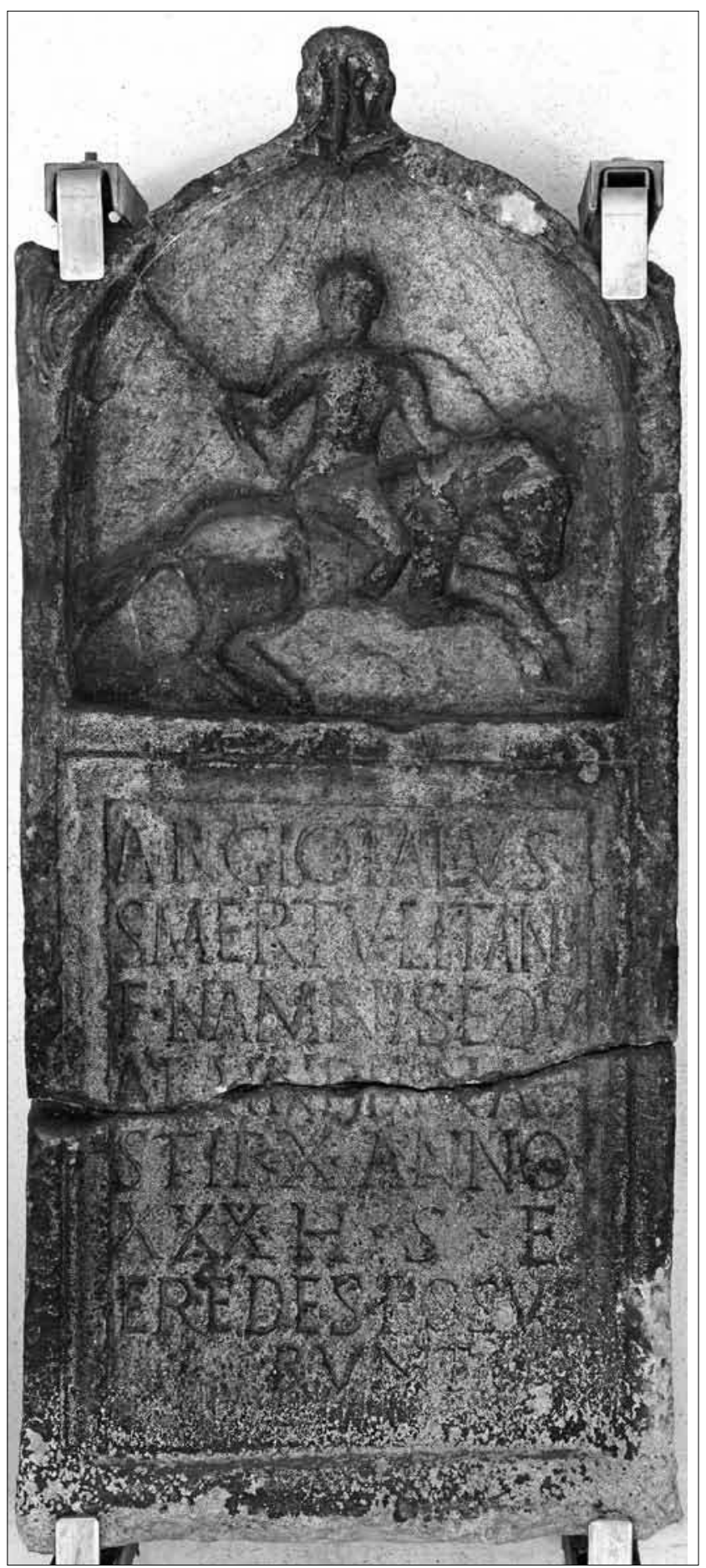

Figure 1 : La stèle d'Argiotalus (Worms, Museum der Stadt Worms im Andreasstift, inv. R 1658. CIL XIII, 6230, Esp. VIII, 6011) (cl. Klaus Baranenko, musée municipal de Worms).

Figure 1: The Argiotalus stela in Worms Municipal Museum.

\author{
ARGIOTALVS $\boldsymbol{\nabla}$ \\ SMERTVLITANI \\ F(ilivs) $\boldsymbol{\nabla}$ NAMNIS $\boldsymbol{\nabla}$ EQV(es) $\boldsymbol{\nabla}$ \\ ALA(e) $\boldsymbol{\nabla}$ INDIANA(e) $\boldsymbol{\nabla}$ \\ STIP(endiorvm) $\boldsymbol{\nabla} \mathrm{X} \boldsymbol{\nabla}$ ANNO(rvm) $\boldsymbol{\nabla}$ \\ $\mathrm{XXX} \boldsymbol{\nabla} \mathrm{H}(\mathrm{ic}) \boldsymbol{\nabla} \mathrm{S}$ (itvs) $\boldsymbol{\nabla} \mathrm{E}(\mathrm{st})$ \\ (h)EREDES $\boldsymbol{\nabla}$ POSVE \\ RVNT
}

c'est-à-dire "Argiotalus, fils de Smertulitanus, Namnète, cavalier de l'aile Indiana, a servi 10 ans, (est décédé à) 30 ans. Il repose ici. Ses héritiers ont érigé [ce monument] ».

Les lignes se terminent par un mot complet ou par une abréviation, sauf l'avant-dernière où le verbe posuerunt est coupé. La graphie gauloise des A et des E n'est pas utilisée : le monument provient de Germanie et du milieu militaire, étroitement soumis aux habitudes romaines. À la $4^{\mathrm{e}}$ ligne, on lit " ala Indiana ", un ablatif employé à la place du génitif plus fréquent "equ(es) alae Indianae "; c'est l'indice d'une datation pré-flavienne de l'épitaphe. La graphie "eredes" pour "heredes", héritiers, n'est pas inconnue. La formule " hic situs est " et l'absence du numéro de l'aile témoignent également d'une datation pré-flavienne (Saddington 1975, p. 184). La formulation du texte, la graphie, les abréviations, la forme des lettres et la ponctuation sont caractéristiques de la première moitié du $\mathrm{I}^{\mathrm{er}}$ siècle.

\section{COMMENTAIRES ET COMPARAISONS}

\section{Forme générale du monument funéraire}

La disposition de la tombe n'est pas connue avec précision. Dans les nécropoles de Worms, les stèles et monuments funéraires étaient entourés d'une haie ou d'un muret déterminant un enclos d'environ deux mètres sur trois. Toutes les tombes du $\mathrm{I}^{\mathrm{er}}$ siècle y sont à incinération, les cendres étant placées dans une urne et enterrées. Dans le sol, l'urne était protégée par un coffre de bois ou de pierre, ou placée dans la logette (loculus) du socle enterré de la stèle (Grünewald 1990a, p. 76-77). Le socle du monument de Maris, à Mayence, mesure 0,66 m de hauteur pour une stèle, complète, haute de 2,10 $\mathrm{m}$ et qui obturait la logette de l'urne.

Le monument funéraire d'Argiotalus est comparable aux stèles du I $^{\text {er }}$ siècle de la Steinhalle du Landesmuseum de Mayence (Selzer 1988). Il fait partie des plus anciennes stèles de cavaliers du " groupe de Mayence " : stèles de Maris, complète (H. 2,10 m + socle de 0,66 m, 1. 0,90 m, ép. 0,28 m : CIL XIII, 7029; Année épigraphique 1959, n 188, p. 130131, n²9, pl. 27 et 1967, 339; Holder 1980, n 672, fig. 


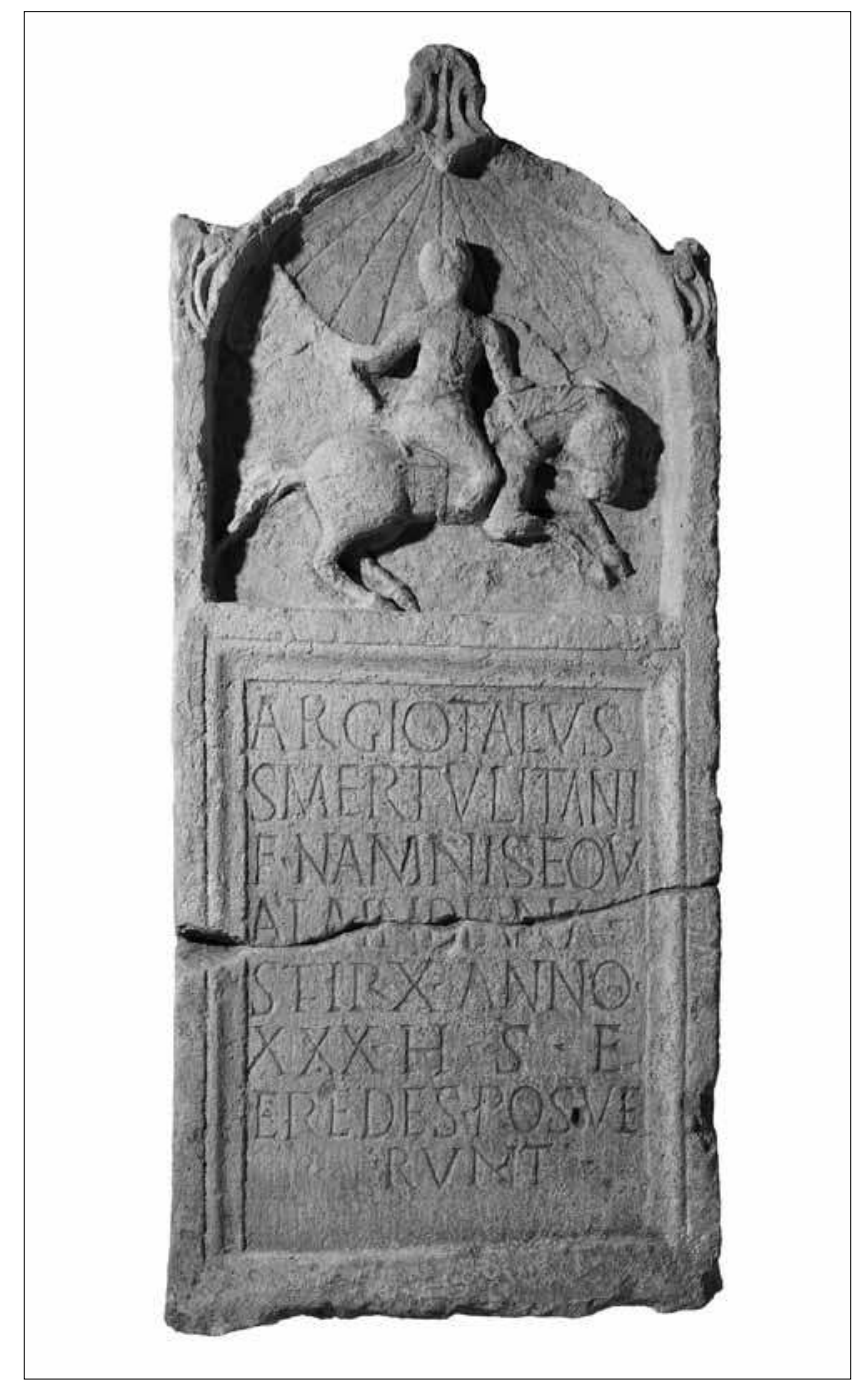

Figure 2 : Stèle d'Argiotalus, moulage de Nantes (cl. H. NeveuDérotrie, musée départemental Dobrée).

Figure 2: Cast of the Argiotalus stela in Dobrée Departmental Museum, Nantes.

3B), ou de C. Romanius Capito (H. 1,63 m, 1. 0,87 m, ép. 0,20 m : Esp. 5852; Boppert 1992, n 31). Mais la stèle d'Argiotalus a des proportions nettement plus élancées malgré la perte de son pied dont la naissance est marquée par un léger évasement de la base sur les quatre faces. La stèle du cavalier Leubius, à Worms, semble avoir conservé l'intégralité de son pied : elle est plus étroite encore et plus élancée (H. 1,92 m, 1. 0,61 m, ép. 0,33 m : CIL XIII, 11709; Esp. 6018; Boppert 1998, $\mathrm{n}^{\circ}$ 51).

La composition à deux registres de la stèle d'Argiotalus relève d'un type de monument funéraire connu dès la première moitié du $\mathrm{I}^{\mathrm{er}}$ siècle de notre ère chez les militaires mais aussi chez les civils de Rhénanie (Feugère 1993, p. 174), dans quelques monuments de cavaliers gallo-romains morts en Bretagne insulaire ${ }^{3}$ et, à Rome, chez les corporis custodes, gardes impériaux montés, sélectionnés parmi les troupes auxiliaires principalement germaniques (stèle de Flavius Proclus, archer arabe de la garde impériale, à Mayence, fin du $\mathrm{I}^{\mathrm{er}}$ siècle : Année épigraphqiue, 1962, 289; Junkelmann 1991, p. 74-75, fig. 33; Speidel 1994, ${ }^{\circ} 1$ ).

Le cadre architectural des stèles funéraires s'inspire de la façade d'un temple et, comme dans les laraires où la divinité apparaît parfois, en épiphanie, debout ou assise entre des antes, des pilastres ou des colonnes comme à la porte de la cella de son temple ${ }^{4}$, le relief funéraire montre moins un portrait qu'une représentation idéalisée du soldat plus ou moins sacralisé, ici figé en héros, vainqueur pour l'éternité. Le brandissement de la javeline et l'attitude du cheval cabré dans la posture de la charge victorieuse évoquent clairement la bravoure et la valeur guerrière du défunt héroïsé. Cette attitude met en valeur l'exploit et suggère l'assurance de la victoire au combat, sans pour autant commémorer une bataille spécifique. Mais elle évoque aussi sans doute la victoire sur la mort et une vague notion de vie d'outre-tombe, pour l'éternité.

\section{Le décor secondaire}

Référence aux niches abritant les divinités (Santrot 2007, p. 81 et 83 , n. 63 et 64 ), la conque marine godronnée qui encadre et protège l'effigie du défunt est assez peu fréquente en Germanie, mais elle peut être observée à Mayence sur une stèle du III $^{\mathrm{e}}$ siècle figurant Mercure domesticus (Esp. 5857; Selzer 28), à Coblence sur un relief à plusieurs divinités (Esp. 6184) et à Cologne sur des autels aux Matrones Axsinginehae et Afliae (Esp. 6401 et 6412). Ce décor est également utilisé à Cologne sur la stèle du soldat $\mathrm{C}$. Gesatus, représenté héroïsé, couché sur le lit de son banquet funéraire (Esp. 6472), et à Mayence sur les stèles du cavalier helvète Rufus (Esp. 5788), de son compatriote Iulius Ingenius (Esp. 5800; Selzer 78, $\mathrm{I}^{\mathrm{er}}$ siècle), de l'aquilifer de la legio XIIII Gemina Cn. Musius (Esp. 5790, av. 43 apr. J.-C.), de l'archer Monimus (Esp. 5861; Selzer 80, $1^{\text {re }}$ moitié du $\mathrm{I}^{\mathrm{er}}$ siècle), du duumvir Licinius Tugnatius Publius (Esp. 5862), et même sur des stèles familiales du $\mathrm{I}^{\mathrm{er}}$ siècle (Selzer 63 et Esp. 5823 = Selzer 105). Il faut peut-être attribuer à la précocité de la stèle d'Argiotalus, l'une des plus anciennes de Rhénanie, la taille anormalement grande de cette conque. Dans les régions rhénanes, ce type de représentation est plus fréquent au $\mathrm{I}^{\text {er }}$ siècle et au début du II siècle (Gabelmann, 1972, p. 65 sq.).

3. Stèles du signifer Flavinus de l'ala Gallorum Petriana, à Corbridge (G.B.), fin du $\mathrm{I}^{\mathrm{er}}$ siècle et de Valerius Genialis, de l'ala I Thracum, à Cirencester (G.-B.), $2^{\mathrm{e}}$ moitié du II ${ }^{\mathrm{e}}$ siècle, originaires de la Gaule et de la Frise; stèle d'un cavalier maure à Neapolis, en Palestine, II siècle (Junkelmann 1991, p. $138-139$, fig. 76 et 78 , et p. $74-74$, fig. 33).

4. Santrot 2003a, p. 73 et $79 ; 2003$ b, p. 208-210. 
Au i ${ }^{\text {er }}$ siècle, la plupart des monuments funéraires de la rive gauche du Rhin étaient ornés d'un décor végétal très simple, probablement d'origine indigène (Hatt, 1970, p. 77-83; 1986, p. 400-405), placé au-dessus de l'inscription, dans le champ du fronton ${ }^{5}$ ou limité à l'arc sommital lorsque la niche en cul-de-four mange le fronton (stèle du cavalier helvète Licinus à Worms, avec arc orné de feuilles imbriquées et écoinçon à fleur à cinq pétales : Esp. 6016). À l'évidence, ces motifs végétaux ne sont pas seulement décoratifs mais ont une valeur symbolique : ils sont porteurs d'une notion de renaissance, de résurrection et d'immortalité, par référence à la végétation qui revit à chaque printemps (Hatt, 1970, p. 77-83). Plus encore, la fleur de carline et les deux rameaux de lierre du fronton de la stèle du boucher C. Vescius, à Bingen, évoquent l'immortalité car la carline séchée se conserve longtemps en s'ouvrant ou se fermant selon l'humidité ambiante et le lierre reste agrippé à son support en gardant ses feuilles vertes tout au long de l'année. Les dauphins affrontés font sans doute référence à leur amitié pour l'homme et à la fidélité que l'on attribuait alors à cet animal (Esp. 6126; Hatt 1970, p. 68-69). Les trois fleurs de pavot (?) du fronton cintré de la stèle de l'archer C. Iulius Hastaius, à Bingen, pourraient bien évoquer le sommeil éternel du défunt (Esp. 6131). Dans le contexte des monuments funéraires gallo-romains, il semble bien que le lierre perde sa seule référence bachique, d'origine grecque, pour acquérir la symbolique, peut-être d'origine indigène, qu’on lui connaît encore : immortalité, attachement, fidélité. À Worms, dans le même contexte que la stèle d'Argiotalus, le monument du cavalier signifer Q. Carminius Ingenuus montre un fronton encore plus dégénéré : le décor d'acanthe des acrotères, quasiment disparus, s'est reporté sur les rampants feuillus du fronton (Esp. 6014). À Mayence, les deux palmes qui encadrent le buste sous coquille de l'archer Monimus ne marquent pas seulement la victoire au combat mais aussi le triomphe sur la mort, tandis que les rinceaux de lierre des rampants du fronton symbolisent l'attachement et l'éternité (Esp. 5861; Selzer 80, $1^{\text {re }}$ moitié du ${ }^{\text {er }}$ siècle).

\section{Le cheval}

En Germanie, la représentation du défunt sur sa stèle funéraire est fréquente chez les légionnaires et les auxiliaires,

5. Fleur à cinq pétales du fronton de la stèle de Peregrinus, à Spire (Esp. 5956; CIL XIII, 6109). Fleur double du fronton de la stèle de C. Vetienius, tubicen de la Ire légion à Cologne (Esp. 6446). Écoinçons et fleuron triangulaire d'acanthe du fronton de la stèle du soldat rhète Firmus à Andernach (Esp. 6207), du cavalier biturige Vellaunus à Bonn (Esp. 6282), du soldat tessinois de la XX légion à Cologne (Esp. 6452). Écoinçons d'acanthe de part et d'autre de la niche d'un cavalier de Worms (Esp. 6033), de la stèle du soldat Annaius à Bingen (Esp. 6125), à Bonn (Esp. 6253, 6255, 6259, 6262), etc. davantage chez les cavaliers que chez les fantassins, mais elle n'est pas systématique loin s'en faut. Argiotalus chevauche une monture de petite stature à la croupe "charnue ", au dos légèrement "concave ", à l'encolure "à bord droit " et "à la crinière épaisse qui retombe à chaque mouvement sur l'épaule droite " comme le préconisent Xénophon et Virgile (Vigneron 1968, p. 6, n. 3). Le cheval semble figuré au troisième temps du galop (ibid., 2, pl. 3) mais ce n'est qu'illusion car le galop du cheval n'a réellement été décomposé et rendu perceptible qu'en 1882 par la chronophotographie d'Étienne Jules Marey. Curieusement, la tête est assez basse pour cette attitude et les oreilles sont rabattues en arrière : le cheval paraît tendu, stressé, prêt à se lâcher brusquement pour une ruade, un saut ou un galop débridé. C'est une posture originale mais bien observée dans l'imagerie funéraire romaine.

Sur les stèles de Worms et de Mayence, les chevaux sont souvent petits par rapport aux cavaliers : à Mayence, les monuments de Maris (Boppert, 1992, p. 29), Togitio (ibid. 32; Esp. 5870), Annauso (Boppert 1992, p. 34; Esp. 5785) ou Andes (Boppert 1992, 35; Esp. 5854) montrent le même type de petits chevaux trapus, d'allure ramassée, que celui d'Argiotalus, tandis que C. Romanius Capito (citoyen romain : Boppert 1992, 31; Esp. 5852), Flavius Proclus (citoyen romain, eques de la garde impériale : Boppert 1992, p. 36) et Petronius Disacentus, fils de Dentubrise (pérégrin : Boppert, 1992, p. 33; Esp. 5786) montent des chevaux de plus grande taille. Cette différence tient-elle à la maladresse du sculpteur, à une convention privilégiant le défunt sur sa monture ou correspond-t-elle à une représentation fidèle des chevaux et à un choix militaire?

La petite taille du cheval d'Argiotalus pourrait en effet n'être qu'une maladresse de sculpteur parmi d'autres ou une convention iconographique privilégiant le défunt. D'ailleurs, la représentation des chevaux sur les stèles funéraires n'est jamais assez précise ni fiable pour permettre de distinguer une race particulière (Dixon et Southern 1992, p. 164). Pourtant, les archéozoologues vérifient peu à peu que les montures des cavaliers gaulois et germains étaient bien issues de races indigènes de petite taille comme le remarquait César : "Les Germains n'importent même pas de chevaux, qui sont la grande passion des Gaulois et qu'ils acquièrent à n'importe quel prix; ils se contentent des chevaux indigènes, qui sont petits et laids, mais qu'ils arrivent à rendre extrêmement résistants grâce à un entraînement quotidien " (BG, IV, 2, p. 98).

La description des méthodes de combat des cavaliers germains dans la Guerre des Gaules montre bien que leurs chevaux n'avaient pas une grande hauteur au garrot: les fantassins accompagnaient la cavalerie en s'agrippant à la crinière des chevaux $(B G, \mathrm{I}, 48,7, \mathrm{p} .41)$ et l'infanterie gauloise de Vercingétorix procédait de même $(B G$, VII, 18,8 , 
p. 222). Au temps de César, les chevaux gaulois ont une hauteur moyenne au garrot inférieure à $1,29 \mathrm{~m}$, avec des hauteurs variant de $1 \mathrm{~m}$ à $1,45 \mathrm{~m}$ (Arbogast et al. 2002, p. 45). La petite taille des chevaux figurés sur les stèles du $\mathrm{I}^{\text {er }}$ siècle en Germanie pourrait ainsi s'expliquer par l'origine des cavaliers : reconnaissables à leur surnom et à leur ethnique, les indigènes devaient probablement être recrutés avec leur propre monture, qu'ils connaissaient bien, et la remonte devait être assurée par l'importation de chevaux issus de leurs régions d'origine ou bien, pour Worms et Mayence et comme le suggère César quatre générations plus tôt, de la Germanie libre toute proche. César laisse entendre qu'il en allait différemment des montures des officiers d'origine italienne, ou de celles des cavaliers romains : "César, qui savait la supériorité de l'ennemi en cavalerie... envoie des messagers au-delà du Rhin, en Germanie... et se fait fournir par eux des cavaliers avec les soldats d'infanterie légère qui sont habitués à combattre dans leurs rangs. À leur arrivée, comme ils avaient des chevaux, petits [ou laids?] et difformes [prava - ou parva? - atque deformia], il les échange contre ceux des tribuns militaires, des autres chevaliers romains, des évocats, et les leur donne "(B.G., VII, 65, p. 258). Sur la taille des chevaux gaulois et romains, voir Arbogast et al. (2002, p. 44-49).

Si cela semble bien montrer que les montures romaines étaient assez généralement plus grandes que celles des Germains, on ne peut aller jusqu’à affirmer que, pour des raisons de prestige, les chevaux des officiers étaient eux-mêmes systématiquement plus grands que ceux de la troupe. C'est pourtant l'hypothèse proposée pour interpréter la différence de taille des ossements de chevaux sur certains sites de l'armée romaine : sur 31 chevaux romains des $\mathrm{I}^{\mathrm{er}}$ et $\mathrm{II}^{\mathrm{e}}$ siècles recueillis dans le camp de Krefeld-Gellep (Grande-Bretagne), seuls deux étaient hauts de 1,14 et 1,24 m. Les autres avaient une hauteur variant entre 1,34 et 1,53 m (Dixon et Southern 1992, p. 166-167). On a songé alors que les chevaux étaient sélectionnés par la taille selon la fonction qui leur était assignée : les plus petits comme animaux du train, ceux de taille moyenne comme montures classiques des cavaliers et les plus grands réservés aux officiers. Pour plausible et logique qu'elle soit, cette hypothèse ne peut être établie actuellement en raison de la faiblesse de la documentation. De même, il n'est pas davantage possible d'étayer suffisamment celle selon laquelle les chevaux des ailes auraient été plus grands que ceux des cohortes equitatae, du fait que les premières étaient plus prestigieuses que les secondes ou auraient eu un rôle tactique différent. D'ailleurs, cela ne serait pas corroboré par la stèle d'Argiotalus ni par celles des autres cavaliers d'ailes mentionnées supra. En réalité, les connaissances actuelles ne permettent pas d'expliquer aujourd'hui la grande variété de la taille des chevaux montés par la cavalerie romaine (Dixon et Southern 1992, p. 166-167 et 170-171). Pour autant, les chevaux des armées de César n'étaient sans doute pas très grands; en effet, une des ruses des Romains était de faire passer pour de la cavalerie les mules du train des équipages et leurs muletiers ( $B G$, VII, 45, p. 242) : certains animaux réservés au transport des bagages devaient donc être d'une taille proche de celle des chevaux de combat. Ainsi, les Romains semblent avoir accordé davantage d'importance aux performances de leurs chevaux qu'à leur taille, même s'il n'est pas improbable que l'on ait réservé des montures exceptionnellement hautes et belles aux officiers supérieurs et aux gardes impériaux car il y avait là une évidente raison de prestige.

La littérature ne donne pas de précisions chiffrées sur la hauteur d'un "petit " ou d'un "grand" cheval mais on observe que les montures antiques atteignaient au plus la taille du poney d'aujourd'hui : d'après Vianney Forest (à paraitre), en Gaule et en Germanie, la hauteur au garrot des chevaux ayant vécu entre 300 av. et 50 apr. J.-C. se situait entre $1 \mathrm{~m}$ et $1,35 \mathrm{~m}$ (contre $1,48 \mathrm{~m}$ en moyenne pour un poney d'aujourd'hui). Elle s'élève, en moyenne, à partir des Flaviens, pour s'établir, de 100 à 400, entre 1,30 et $1,55 \mathrm{~m}$ (qu'il faut comparer au $1,80 \mathrm{~m}$ des chevaux de selle d'aujourd'hui $\left.{ }^{6}\right)$. Cette évolution doit sans doute moins à l'apport de races méditerranéennes et orientales, de plus grande taille semble-t-il, qu'à l'amélioration du cheptel indigène, sans doute principalement par l'apport d'une alimentation choisie, par la maîtrise de l'âge de la mise à la reproduction et, mais c'est moins sûr, par la sélection. C'est ce qui apparaît sur le site de Chelmsford (GrandeBretagne), bien qu'on ne sache si l'on doit attribuer la croissance des chevaux sur quatre siècles à une sélection de races ou à l'apport de races extérieures (Dixon et Southern 1992, p. 171-172). C'est également vers le milieu du $\mathrm{I}^{\mathrm{er}}$ siècle que se fait l'évolution des métatarses des bovins qui, de " petits" et "moyens " auparavant, deviennent "grands " et " très grands » ensuite (Forest et Rodet-Belarbi 2002, p. 284) : la corpulence des bovins augmente en effet d'environ $15 \%$ à partir du milieu du i ${ }^{\text {er }}$ siècle (ibid., p. 294-296).

\section{Le harnachement (tableau 1)}

Fidèlement sculpté, le harnachement du cheval d'Argiotalus est du modèle sans phalères, le plus fréquent sur les stèles

6. Vianney Forest, docteur vétérinaire et archéozoologue de l'INRAP, précise que, dans cette population de taille modeste, on rencontre aussi des " géants " de 1,50 m au garrot, peut-être venus de l'est de l'Europe où la moyenne était de $1,37 \mathrm{~m}$ (entre 1,21 et $1,50 \mathrm{~m}$ ). Ces chevaux de plus grande taille sont peut-être issus de sélections mais surtout de soins particuliers appliqués au cours de leur croissance (castration, alimentation, etc.). "La taille des chevaux romains oscille entre 145 et $155 \mathrm{~cm}$, pouvant atteindre et dépasser les $160 \mathrm{~cm}$ de hauteur " (Audouin-Rouzeau, 1995, p. 81-82). 


\begin{tabular}{|c|c|c|c|c|c|c|c|c|}
\hline CAVALIERS & $\begin{array}{c}\text { Unité } \\
\text { Datation }\end{array}$ & $\begin{array}{l}\text { Harnais } \\
\text { de tête }\end{array}$ & $\begin{array}{c}\text { Courroies } \\
\text { du poitrail }\end{array}$ & $\begin{array}{l}\text { Courroies } \\
\text { du flanc } \\
\text { et de queue }\end{array}$ & $\begin{array}{l}\text { Pendants } \\
\text { de lanière }\end{array}$ & $\begin{array}{c}\text { Selle et } \\
\text { tapis de selle }\end{array}$ & $\begin{array}{l}\text { Anneaux de } \\
\text { régulation }\end{array}$ & Phalères \\
\hline ARGIOTALUS & $\begin{array}{l}\text { Cavalier d'aile } \\
\text { Worms } \\
1^{\text {ère }} \text { moitié du I }{ }^{\mathrm{er}} \mathrm{s} \text {. }\end{array}$ & $\begin{array}{l}\text { Muserolle } \\
\text { Courroie de } \\
\text { chanfrein à } \\
\text { double bourrelet } \\
\text { Porte-mors } \\
\text { Frontal } \\
\text { Sous-gorge }\end{array}$ & $\begin{array}{l}\text { Bricole et } \\
\text { martingale }\end{array}$ & $\begin{array}{l}\text { Courroie de } \\
\text { queue droite, } \\
\text { reliée à l'anneau } \\
\text { de séparation }\end{array}$ & $\begin{array}{l}\text { Sur la jambe } \\
\text { avant }\end{array}$ & $\begin{array}{l}\text { Selle petite et } \\
\text { plate } \\
\text { Arçon arrière } \\
\text { saillant } \\
\text { Chabraque } \\
\text { à croisillons } \\
\text { losangés }\end{array}$ & $\begin{array}{l}\text { Anneau } \\
\text { d'articulation } \\
\text { reliant la selle } \\
\text { à la sangle } \\
\text { arrière. }\end{array}$ & \\
\hline $\begin{array}{l}\text { ANDES } \\
\text { Selzer 1988, } \\
\mathrm{n}^{\circ} 88 \\
\text { Boppert 1992, } \\
\mathrm{n}^{\circ} 35\end{array}$ & $\begin{array}{l}\text { Cavalier d'aile } \\
\text { Mayence } \\
2^{\text {e }} \text { moitié } \mathrm{I}^{\mathrm{er}} \mathrm{s} \text {. }\end{array}$ & $\begin{array}{l}\text { Muserolle avec } \\
\text { mors relié au } \\
\text { porte-mors et } \\
\text { à la rêne } \\
\text { Sangle de joue } \\
\text { parallèle au } \\
\text { porte-mors } \\
\text { Têtière et frontal } \\
\text { Chanfrein (?) }\end{array}$ & $\begin{array}{l}\text { Bricole reliée } \\
\text { à la selle par deux } \\
\text { phalères } \\
\text { Ornement en } \\
\text { bandes posé sur } \\
\text { la base du cou du } \\
\text { cheval }\end{array}$ & $\begin{array}{l}\text { Courroie de } \\
\text { queue reliée à } \\
\text { la selle } \\
\text { par deux anneaux. } \\
\text { La base de la } \\
\text { queue semble } \\
\text { enroulée d'une } \\
\text { courroie }\end{array}$ & $\begin{array}{l}\text { Sur la jambe } \\
\text { arrière }\end{array}$ & $\begin{array}{l}\text { Selle à arçons } \\
\text { avant et arrière } \\
\\
\text { Tapis de selle } \\
\text { à bandes } \\
\text { ornementales } \\
\text { flottantes }\end{array}$ & $\begin{array}{l}2 \text { anneaux } \\
\text { sur la croupe } \\
\text { du cheval, } \\
\text { à la place de } \\
\text { phalères }\end{array}$ & $\begin{array}{l}2 \text { phalères : } \\
\text { sur le poitrail } \\
\text { et sur l'épaule }\end{array}$ \\
\hline $\begin{array}{l}\text { CAIUS } \\
\text { ROMANIUS } \\
\text { CAPITO } \\
\text { Selzer } 1988, \\
n^{\circ} 87 \\
\text { Boppert } 1992, \\
n^{\circ} 31\end{array}$ & $\begin{array}{l}\text { Cavalier d'aile } \\
\text { Mayence } \\
\mathrm{I}^{\mathrm{er}} \text { siècle }\end{array}$ & $\begin{array}{l}\text { Muserolle avec } \\
\text { mors relié à la } \\
\text { rêne et à un } \\
\text { porte-mors } \\
\text { double }\end{array}$ & $\begin{array}{l}\text { Bricole reliée } \\
\text { à l'avant de la } \\
\text { selle par une } \\
\text { phalère }\end{array}$ & $\begin{array}{l}\text { Courroie de } \\
\text { queue reliée à } \\
\text { l'arrière de la selle } \\
\text { par une phalère }\end{array}$ & $\begin{array}{l}\text { Sur les jambes } \\
\text { avant } \\
\text { et arrière }\end{array}$ & 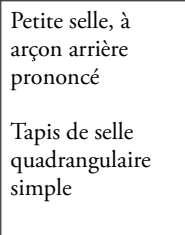 & $\begin{array}{l}\text { Anneau } \\
\text { au niveau de } \\
\text { la bride } \\
\text { Anneau sur la } \\
\text { tête, à l'extrémité } \\
\text { supérieure du } \\
\text { porte-mors }\end{array}$ & $\begin{array}{l}3 \text { phalères } \\
\text { stylisées } \\
\text { symétriques : } \\
\text { sur l'épaule, } \\
\text { sur le poitrail } \\
\text { et à l'arrière }\end{array}$ \\
\hline $\begin{array}{l}\text { MARIS } \\
\text { Selzer 1988, } \\
n^{\circ} 91 \\
\text { Boppert 1992, } \\
n^{\circ} 29 \\
\text { (surface usée) }\end{array}$ & $\begin{array}{l}\text { Cavalier d'aile } \\
\text { Mayence } \\
\text { Milieu du I }{ }^{\mathrm{er}} \mathrm{s} .\end{array}$ & $\begin{array}{l}\text { Muserolle } \\
\text { Porte-mors } \\
\text { Sous-gorge } \\
\text { Chanfrein (?) } \\
\text { Rêne }\end{array}$ & & Courroie de flanc & & & & \\
\hline $\begin{array}{l}\text { ANNAUSO } \\
\text { Selzer 1988, } \\
n^{\circ} 86 \\
\text { Boppert 1992, } \\
n^{\circ} 34 \\
\text { (surface usée) }\end{array}$ & $\begin{array}{l}\text { Cavalier d'aile } \\
\text { Mayence } \\
2^{\text {e }} \text { moitié } \mathrm{I}^{\mathrm{er}} \mathrm{s} \text {. }\end{array}$ & Muserolle & & & & $\begin{array}{l}\text { Bords de selle } \\
\text { visibles à l'avant } \\
\text { et surtout à } \\
\text { l'arrière }\end{array}$ & & \\
\hline $\begin{array}{l}\text { PETRONIUS } \\
\text { DISACENTUS } \\
\text { Selzer 1988, } \\
\mathrm{n}^{\circ} 89 \\
\text { Boppert 1992, } \\
\mathrm{n}^{\circ} 33\end{array}$ & $\begin{array}{l}\begin{array}{l}\text { Cavalier de } \\
\text { cohorte }\end{array} \\
\text { Mayence } \\
\text { Milieu du i }{ }^{\text {er }} \text { s. }\end{array}$ & $\begin{array}{l}\text { Muserolle avec } \\
\text { mors relié au } \\
\text { porte-mors } \\
\text { et à la rêne } \\
\text { Rêne à plusieurs } \\
\text { bandelettes } \\
\text { de cuir (?) }\end{array}$ & $\begin{array}{l}\text { Bricole reliée } \\
\text { à la selle par une } \\
\text { phalère }\end{array}$ & & $\begin{array}{l}\text { Sur les jambes } \\
\text { avant } \\
\text { et arrière }\end{array}$ & $\begin{array}{l}\begin{array}{l}\text { Selle à haut arçon } \\
\text { arrière }\end{array} \\
\text { Tapis de selle à } \\
\text { longues lanières } \\
\text { pendantes }\end{array}$ & $\begin{array}{l}2 \text { phalères } \\
\text { ornementales } \\
\text { en forme de } \\
\text { fleurs à l'arrière } \\
\text { et à l'avant }\end{array}$ & \\
\hline $\begin{array}{l}\text { TITUS FLAVIUS } \\
\text { BASSUS } \\
\text { Dixon et } \\
\text { Southern } \\
1997 \text {, fig. } 7\end{array}$ & $\begin{array}{l}\text { Cavalier d'aile } \\
\text { Cologne } \\
\text { Fin du I }{ }^{\text {er }} \mathrm{s} \text {. }\end{array}$ & $\begin{array}{l}\text { Muserolle avec } \\
\text { mors relié par un } \\
\text { anneau à la rêne } \\
\text { Porte-mors relié } \\
\text { par un anneau } \\
\text { au frontal et } \\
\text { à la sous-gorge } \\
\text { Long collier } \\
\text { décoratif sur } \\
\text { l'encolure }\end{array}$ & $\begin{array}{l}\text { Bricole reliée } \\
\text { à la phalère puis } \\
\text { à la selle par une } \\
\text { double sangle } \\
\text { Bande } \\
\text { ornementale sur } \\
\text { le poitrail }\end{array}$ & $\begin{array}{l}\text { Courroie de } \\
\text { queue reliée à la } \\
\text { phalère puis à } \\
\text { la selle par une } \\
\text { sangle double }\end{array}$ & $\begin{array}{l}\text { Sur la jambe } \\
\text { arrière }\end{array}$ & $\begin{array}{l}\begin{array}{l}\text { Selle aux bords } \\
\text { avant et arrière } \\
\text { saillants (assise } \\
\text { assurée) }\end{array} \\
\text { Tapis de selle } \\
\text { identifiable sous } \\
\text { l'arrondi de la } \\
\text { selle }\end{array}$ & & $\begin{array}{l}\text { Phalères } \\
\text { sur l'épaule } \\
\text { et la cuisse }\end{array}$ \\
\hline $\begin{array}{l}\text { VONATORIX } \\
\text { Junkelmann } \\
\text { 1991, fig. } 26 \\
\text { Dixon et } \\
\text { Southern } 1997 \text {, } \\
\text { fig. } 8\end{array}$ & $\begin{array}{l}\text { Cavalier d'aile } \\
\text { Bonn } \\
\text { Milieu }{ }^{\mathrm{er}} \mathrm{s} . \\
(40-69 \mathrm{AD})\end{array}$ & $\begin{array}{l}\text { Muserolle avec } \\
\text { mors et anneau } \\
\text { Porte-mors } \\
\text { Frontal décoré } \\
\text { Sous-gorge } \\
\text { Rêne reliée } \\
\text { au mors }\end{array}$ & $\begin{array}{l}\text { Martingale } \\
\text { passant sous le } \\
\text { poitrail }\end{array}$ & $\begin{array}{l}\text { Courroie de } \\
\text { queue très visible, } \\
\text { reliée à la phalère } \\
\text { de cuisse }\end{array}$ & $\begin{array}{l}\text { Sur les jambes } \\
\text { avant } \\
\text { et arrière }\end{array}$ & 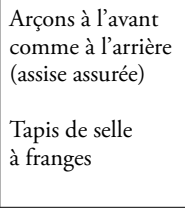 & $\begin{array}{l}\text { Non visibles: } \\
\text { sous les phalères? }\end{array}$ & $\begin{array}{l}\text { Multiples phalères } \\
\text { stylisées au niveau } \\
\text { de la muserolle, } \\
\text { du frontal, de la } \\
\text { courroie arrière, } \\
\text { de l'épaule, } \\
\text { du poitrail }\end{array}$ \\
\hline $\begin{array}{l}\text { TOGITIO } \\
\text { Junkelmann } \\
\text { 1991, fig. } 55 \\
\text { Boppert } 1992 \text {, } \\
\text { n}^{\circ} 32\end{array}$ & $\begin{array}{l}\text { Cavalier auxiliaire } \\
\text { Mannheim } \\
\text { milieu du }{ }^{\text {er }} \mathrm{s} \text {. }\end{array}$ & $\begin{array}{l}\text { Muserolle } \\
\text { Rêne } \\
\text { Sous-gorge? } \\
\text { Frontal }\end{array}$ & $\begin{array}{l}\text { Bricole reliée } \\
\text { à la phalère de } \\
\text { l'épaule }\end{array}$ & $\begin{array}{l}\text { Courroie de } \\
\text { queue reliée à } \\
\text { la phalère de la } \\
\text { cuisse et à l'arrière } \\
\text { de la selle }\end{array}$ & $\begin{array}{l}\text { Sur les jambes } \\
\text { avant } \\
\text { et arrière }\end{array}$ & $\begin{array}{l}\text { Pas de selle } \\
\text { visible, peut-être } \\
\text { pommeau et bord } \\
\text { avant? } \\
\text { Pas de tapis de } \\
\text { selle visible }\end{array}$ & & $\begin{array}{l}2 \text { phalères } \\
\text { stylisées : } \\
\text { sur la cuisse } \\
\text { et sur l'épaule }\end{array}$ \\
\hline
\end{tabular}




\begin{tabular}{|c|c|c|c|c|c|c|c|c|}
\hline $\begin{array}{l}\text { FLAVINUS } \\
\text { Junkelmann } \\
\text { 1991, fig. } 76\end{array}$ & $\begin{array}{l}\text { Signifer d'aile } \\
\text { Corbridge } \\
\text { Fin du } \mathrm{I}^{\mathrm{er}} \mathrm{s} .\end{array}$ & $\begin{array}{l}\text { Large muserolle } \\
\text { Frontal } \\
\text { Sangle de joue } \\
\text { avec phalère (?) } \\
\text { Rêne }\end{array}$ & $\begin{array}{l}\text { Bricole reliée } \\
\text { à la selle et la } \\
\text { sangle de flanc. }\end{array}$ & $\begin{array}{l}\text { Sangle de flanc } \\
\text { reliée } \\
\text { à la selle } \\
\text { Courroie de } \\
\text { queue reliée à la } \\
\text { précédente par } \\
\text { un anneau }\end{array}$ & $\begin{array}{l}\text { Sur les jambes } \\
\text { avant } \\
\text { et arrière }\end{array}$ & $\begin{array}{l}\begin{array}{l}\text { Selle à bord plat } \\
\text { à l'avant et à } \\
\text { l'arrière }\end{array} \\
\text { Tapis de selle } \\
\text { à bords ourlés }\end{array}$ & \begin{tabular}{|l}
2 anneaux \\
d'articulation : \\
- sur l'épaule, \\
reliant la sangle \\
du poitrail à la \\
selle, \\
- sur la cuisse, \\
reliant la selle \\
à la sangle de \\
queue
\end{tabular} & $\begin{array}{l}\text { Phalère } \\
\text { sur la joue (?) }\end{array}$ \\
\hline $\begin{array}{l}\text { VALERIUS } \\
\text { GENIALIS } \\
\\
\text { Junkelmann } \\
\text { 1991, fig. } 78\end{array}$ & $\begin{array}{l}\text { Cavalier d'aile } \\
\text { Circencester } \\
2^{e} \text { moitié } \mathrm{II}^{\mathrm{e}} \mathrm{s} .\end{array}$ & $\begin{array}{l}\text { Large muserolle } \\
\text { Sangle de joue } \\
\text { Frontal } \\
\text { Fuseau de } \\
\text { plusieurs rênes } \\
\text { fines }\end{array}$ & Bricole peu visible & \begin{tabular}{|l|} 
Sangle de flanc \\
reliée \\
à la selle \\
Courroie de \\
queue reliée à la \\
sangle par une \\
phalère \\
\end{tabular} & $\begin{array}{l}\text { Larges pendants } \\
\text { sur la cuisse } \\
\text { (non visibles à } \\
\text { l'avant) }\end{array}$ & \begin{tabular}{|l} 
Selle à arçon \\
arrière \\
Tapis de selle \\
à franges
\end{tabular} & & $\begin{array}{l}\text { Phalère reliant } \\
\text { sangle de flanc } \\
\text { et sangle de queue }\end{array}$ \\
\hline $\begin{array}{l}\text { CARMINIUS } \\
\text { INGENUUS } \\
\text { Junkelmann } \\
1991 \text {, fig. } 79\end{array}$ & $\begin{array}{l}\text { Signifer d'aile } \\
\text { Worms } \\
\text { Milieu du } \mathrm{r}^{\mathrm{er}} \mathrm{s} .\end{array}$ & \begin{tabular}{|l} 
Sous-gorge \\
Muserolle \\
Sangle de joue \\
Frontal avec \\
phalère \\
Rêne
\end{tabular} & $\begin{array}{l}\text { Bricole avec } \\
\text { phalère, reliée à } \\
\text { la selle par une } 2^{\text {e }} \\
\text { phalère }\end{array}$ & \begin{tabular}{|l|} 
Courroie de \\
queue reliée à la \\
phalère puis à la \\
selle par l'anneau \\
d'articulation
\end{tabular} & $\begin{array}{l}\text { Sur la jambe } \\
\text { avant } \\
\text { Moins visible sur } \\
\text { la jambe arrière }\end{array}$ & $\begin{array}{l}\text { La selle n'est pas } \\
\text { visible } \\
\text { (sauf bord avant } \\
\text { épais ?) }\end{array}$ & \begin{tabular}{|l|} 
Petit anneau \\
d'articulation \\
à l'arrière de la \\
selle, au-dessus de \\
la phalère
\end{tabular} & \begin{tabular}{|l} 
Plusieurs phalè- \\
res stylisées : entre \\
le frontal et la \\
sangle de joue, sur \\
le poitrail, \\
l'épaule et la \\
cuisse
\end{tabular} \\
\hline $\begin{array}{l}\text { VELLAUNUS } \\
\text { Junkelmann } \\
\text { 1991, fig. } 81\end{array}$ & $\begin{array}{l}\text { Cavalier d'aile } \\
\text { Bonn } \\
\text { Milieu du } \mathrm{I}^{\mathrm{er}} \mathrm{s} .\end{array}$ & \begin{tabular}{|l} 
Muserolle avec \\
mors \\
Sangle de joue \\
Frontal \\
Rêne \\
\end{tabular} & \begin{tabular}{|l|} 
Bricole à phalère \\
reliée \\
à la selle par \\
la phalère \\
d'épaule
\end{tabular} & $\begin{array}{l}\text { Courroie de } \\
\text { queue reliée à la } \\
\text { selle, peut-être par } \\
\text { une phalère (?) }\end{array}$ & $\begin{array}{l}\text { Pendant de } \\
\text { lanière large sur } \\
\text { l'épaule }\end{array}$ & \begin{tabular}{|l|}
$\begin{array}{l}\text { Selle à arçons } \\
\text { élevés }\end{array}$ \\
Tapis de selle \\
quadrangulaire
\end{tabular} & & $\begin{array}{l}\text { Phalères de } \\
\text { poitrail et } \\
\text { d'épaule. } \\
\text { Phalère de cuisse } \\
\text { cachée? }\end{array}$ \\
\hline $\begin{array}{l}\text { FLAVIUS } \\
\text { PROCLUS } \\
\text { Speidel 1994, } \\
\text { fig. 1 } \\
\text { Dixon et } \\
\text { Southern 1997, } \\
\text { fig. } 23 \\
\text { Selzer 1988, } \\
\text { n }^{\circ} 90 \\
\text { Boppert 1992, } \\
n^{\circ} 36\end{array}$ & $\begin{array}{l}\text { Eques Singularis } \\
\text { Augusti } \\
\text { Archer } \\
\text { Mayence } \\
\text { Fin du } \mathrm{I}^{\mathrm{er}} \mathrm{s} \text {. }\end{array}$ & $\begin{array}{l}\text { Frontal } \\
\text { peu visible } \\
\text { Rêne reliée au } \\
\text { mors }\end{array}$ & & & $\begin{array}{l}\text { Peut-être sur la } \\
\text { cuisse (?) }\end{array}$ & \begin{tabular}{|l} 
Selle peu visible : \\
pommeau ou \\
saillant avant \\
Tapis de selle \\
identifiable à \\
l'avant
\end{tabular} & & \begin{tabular}{|l} 
Peut-être une \\
phalère \\
à l'arrière \\
(à moins qu'il \\
ne s'agisse d'un \\
anneau ?)
\end{tabular} \\
\hline $\begin{array}{l}\text { GAIUS MARIUS } \\
\text { Junkelmann } \\
1991 \text {, fig. } 25\end{array}$ & \begin{tabular}{|l} 
Cavalier \\
légionnaire \\
Bonn \\
Milieu du $\mathrm{r}^{\mathrm{er}} \mathrm{s}$. \\
\end{tabular} & \begin{tabular}{|l} 
Muserole \\
Bride \\
Frontal et \\
sous-gorge peu \\
visibles
\end{tabular} & $\begin{array}{l}\text { Martingale reliée } \\
\text { à l'avant de la } \\
\text { selle }\end{array}$ & $\begin{array}{l}\text { Simple courroie } \\
\text { de queue passant } \\
\text { sur le côté de la } \\
\text { selle et rejoignant } \\
\text { la bricole }\end{array}$ & & $\begin{array}{l}\begin{array}{l}\text { Pas de tapis de } \\
\text { selle visible }\end{array} \\
\text { Petite selle à bords } \\
\text { plats à l'avant et à } \\
\text { l'arrière }\end{array}$ & Pas d'anneau & \\
\hline
\end{tabular}

\begin{tabular}{|c|c|c|c|c|c|c|c|c|}
\hline $\begin{array}{l}\text { CHEVAUX EN } \\
\text { PARADE } \\
\text { non montés }\end{array}$ & $\begin{array}{c}\text { Unité } \\
\text { Datation }\end{array}$ & $\begin{array}{l}\text { Harnais } \\
\quad \text { de } \\
\text { tête }\end{array}$ & $\begin{array}{c}\text { Courroies } \\
\text { du poitrail }\end{array}$ & $\begin{array}{l}\text { Courroies } \\
\text { du flanc } \\
\text { et de queue }\end{array}$ & $\begin{array}{l}\text { Pendants de } \\
\quad \text { lanière }\end{array}$ & $\begin{array}{c}\text { Selle et } \\
\text { tapis de selle }\end{array}$ & $\begin{array}{c}\text { Anneaux } \\
\text { de } \\
\text { régulation }\end{array}$ & Phalères \\
\hline $\begin{array}{l}\text { VIRUNO } \\
\text { Speidel 1994, } \\
\text { fig. } 2\end{array}$ & $\begin{array}{l}\text { Eques Singularis } \\
\text { Augusti } \\
\text { Rome }\end{array}$ & & & & & $\begin{array}{l}\text { Long tapis } \\
\text { (sans selle) destiné } \\
\text { à la parade }\end{array}$ & & \\
\hline $\begin{array}{l}\text { COLONNE } \\
\text { AURÉLIENNE } \\
\text { Speidel 1994, } \\
\text { fig. } 3\end{array}$ & $\begin{array}{l}\text { Eques Singularis } \\
\text { Augusti } \\
\text { Rome }\end{array}$ & $\begin{array}{l}\text { Muserolle } \\
\text { Rêne reliée au } \\
\text { mors }\end{array}$ & & & & $\begin{array}{l}\text { Long tapis sans } \\
\text { selle : parade } \\
\text { lors de la revue } \\
\text { des troupes par } \\
\text { l'empereur }\end{array}$ & & \\
\hline $\begin{array}{l}\text { OCLATIUS } \\
\text { Junkelmann } \\
\text { 1991, fig. } 77\end{array}$ & $\begin{array}{l}\text { Signifer d'aile } \\
\text { Neuss } \\
\text { Vers } 100 \\
\text { apr. J.-C. }\end{array}$ & \begin{tabular}{|l|} 
Bride tirée \\
par le calo
\end{tabular} & & & & \begin{tabular}{|l}
$\begin{array}{l}\text { Long tapis } \\
\text { (sans selle) }\end{array}$ \\
\end{tabular} & & \\
\hline
\end{tabular}

Tableau 1 : Harnachements militaires représentés sur les monuments évoqués dans le texte.

Table 1: Military harnesses shown on monuments mentionned in text. 
d'auxiliaires (Forest à paraître). Les phalères ornent indistinctement les chevaux des auxiliaires et des légionnaires, et ne semblent donc pas relever d'un type d'unité particulier. Elles ornent ainsi plusieurs stèles de cavaliers auxiliaires, simples cavaliers ou sous-officiers, comme Andes, Vonatorix, Togitio, Flavinius (signifer), Valerius Genialis (signifer), Carminius Ingenuus (signifer), Vellaunus, etc. (Junkelmann 1991, fig. 26, 55, 76, 78, 79, 81). Au contraire, les montures des cavaliers légionnaires Flavius Proclus et Gaius Marius ne portent aucune phalère (Speidel 1994, $\mathrm{n}^{\circ} 1$; Junkelmann 1991, fig. 25).

Du harnais de tête, on distingue la muserolle et la courroie de chanfrein à double bourrelet, moins distinctement le porte-mors et le frontal, épaufrés, et peut-être une sousgorge. La forme du mors n'est plus perceptible. Du harnachement, on reconnaît les courroies de la queue et de l'arrière-main (la croupière), celles du poitrail (la bricole et la martingale) et celles du flanc (sangles) ainsi que, sur la jambe avant, un pendant de lanière qui ne figure pas sur la cuisse arrière $^{7}$. La rêne droite, partiellement épaufrée, est posée sur l'encolure.

La petite selle, très plate mais à l'arçon arrière légèrement saillant (Vigneron 1968, p. 83, n. 9, et 2, pl. 29, e), est posée sur une couverture - ou un tapis de selle (chabraque) - ornée de croisillons losangés; elle semble maintenue par deux sangles pour protéger le ventre du cheval. À l'arrière-main, la courroie de croupe est reliée par un anneau au bord arrière droit de la selle et à la sangle arrière. Le tapis de selle est orné de motifs originaux, aujourd'hui peu perceptibles mais jadis sans doute très colorés, alors que, habituellement, ce n'est qu'une simple pièce de tissu rectangulaire sans décor apparent, au mieux bordé d'un ourlet (stèle de Flavinus : Junkelmann 1991, fig. 76), ou d'une frange (stèles de Vonatorix et de Valerius Genialis : ibid., fig. 26 et 78).

À l'arrière-main, la courroie de croupe est reliée par un anneau au bord arrière droit de la selle et à la sangle arrière. Le harnachement du petit cheval d'Argiotalus montre en particulier les anneaux de réglage des sangles et le chanfrein, rarement visibles ou représentés.

Le harnais et les différentes sangles des montures antiques sont semblables, quelle que soit l'unité concernée : le harnais le plus simple est constitué d'une muserolle et d'un frontal relié à une têtière. Le mors est relié aux rênes et au portemors, parfois à l'aide d'un anneau. On observe également une sous-gorge et plus rarement un chanfrein. Le harnachement de la monture d'Argiotalus correspond au harnachement "standard » des cavaliers légionnaires et auxiliaires observé sur les stèles funéraires du $\mathrm{I}^{\mathrm{er}}$ siècle.

7. Courroies tombantes lisibles sur la restitution colorée de la stèle de Silius, à Mayence (Cüppers 1990, pl. coul. 4) ou sur la stèle de Cantaber (Esp. 5785; Boppert 1992, n 30. Cf. Feugère 1993, p. 174-180).

\section{Le cavalier et son armement}

Argiotalus est figuré sur son cheval au galop (ou cabré), chargeant un ennemi invisible ${ }^{8}$, vers la droite, côté favorable. À défaut de réalisme - la perspective n'est guère de mise dans ce type de relief - mais pour donner l'impression de mouvement, le bassin, la cuisse et la jambe droites sont de profil tandis que le torse semble de face. C'est l'attitude, ici inversée, que l'on constate sur un cavalier de la frise occidentale du Parthénon qui, lui, regarde bien en arrière (Vigneron 1968, 2, pl. 3).

Imberbe, il semble se retourner (une barbe préciserait la direction du regard malgré l'érosion du relief). Pourtant, cette impression n'est due qu'à l'usure de la pierre et à un défaut d'implantation du cou, trop déporté à gauche si le torse est bien représenté de face, mais assez bien placé si le cou et la tête sont de profil, car, à la différence du cavalier de la frise occidentale du Parthénon, l'homme ne se retourne jamais dans l'iconographie du type. Sur les stèles adoptant la même iconographie, le cavalier a la tête de profil, ou de trois-quarts, et regarde l'ennemi qu'il s'apprête à transpercer. L'érosion de la tête ne permet pas ici de vérifier la présence du casque dont sont habituellement dotés les cavaliers (Feugère 1993, p. 181-184) : seul l'élargissement du crâne, très arrondi et peut-être surdimensionné, pourrait éventuellement plaider en faveur de la présence d'un casque à timbre sphérique ${ }^{9}$.

Comme C. Romanius Capito à Mayence (Esp. 5852), Argiotalus chevauchait pieds nus sans doute, les jambes

8. Mieux conservée, la stèle de C. Romanius Capito, à Mayence, montre que le cavalier chevauche jambes et pieds nus, que son cheval porte un harnachement plus complet, enrichi d'imposantes phalères métalliques, et que, accompagné au combat par son valet d'armes, le calo, qui tient à sa disposition deux courtes lances ou javelines de rechange, il charge et s'apprête à percer de sa lance un ennemi étendu au sol entre les jambes de sa monture tandis que le cheval cabré brise la tête ennemie de ses sabots. Le piétinement de l'ennemi vaincu est particulièrement symbolique (Esp. 5852; Boppert 1992, n 31).

9. Des stèles du I $^{\text {er }}$ siècle, à Mayence, présentent assez fréquemment un casque à calotte figurant une chevelure ondulée et bouclée : Annauso (Selzer 86; Junkelmann 1991, fig. 44), C. Romanius Capito (Selzer 87), Andes (Selzer 88) et Togitio (Junkelmann 1991, fig. 55). Plus rares et tardifs sont les casques de parade : Valerius Genialis à Cirencester (G.-B.), $2^{\mathrm{e}}$ moitié du II ${ }^{\mathrm{e}}$ siècle (Selzer 78). Mais certains cavaliers sont représentés tête nue, comme Vonatorix, cavalier de l'ala Longiniana stationnée à Bonn, disparu au milieu du Ir siècle (Dixon et Southern 1992, fig. 9; Junkelmann 1991, fig. 26), ou le signifer de l'Ala I Hispanorum de Worms, Carminius Ingenuus, citoyen romain, contemporain d'Argiotalus (Junkelmann 1991, fig. 79). Si Argiotalus était casqué, le timbre de son casque devait être une simple calotte métallique avec paragnathides et couvre-nuque; un casque à chevelure comme ceux de ses contemporains de Mayence est également possible (Dixon et Southern 1992, p. 35; Robinson 1975, p. 89 sq.), à comparer aux casques de fer ou de bronze à calotte lisse, trouvés dans le Rhin à Mayence (types Haguenau et Weisenau), courants au i ${ }^{\text {er }}$ siècle (Klein 2003, p. 30-35). 
ramenées sous le ventre du cheval pour s'assurer une bonne assiette mais aussi pour le diriger puisqu'il ne semble pas tenir les rênes, habituellement dans la main gauche mais ici posées sur l'encolure. Paradoxalement, les cavaliers chaussés de sandales semblent rares (à Mayence : Cantaber : Esp. 5784; Boppert 1992, $\mathrm{n}^{\circ} 30$; Andes, $2^{\mathrm{e}}$ moitié du I ${ }^{\mathrm{er}}$ siècle : Selzer 1988, fig. 45; Romanius : ibid., fig. 48; etc.) et, sans que cela soit constant, bien des fantassins en armes, légionnaires ou auxiliaires, sont également figurés les pieds nus (Esp. 5792, 5835; Boppert 1992, nº 7 à 10. A Mayence, à la seconde moitié du I ${ }^{\text {er }}$ siècle, Q. Luccius Faustrus : Selzer 1988, fig. 17; Genialis : ibid., fig. 27; P. Flavoleius Cordus : ibid., $\mathrm{n}^{\circ} 26$; etc.). Peut-être les lanières des sandales, indispensable équipement du militaire, étaient-elles détaillées par la peinture? Ou bien la nudité du pied était-elle considérée comme un signe supplémentaire d'héroïsation et d'assimilation à la divinité? Ici, le pied a tout bonnement disparu.

L'armement défensif n'est guère perceptible : le probable casque est effacé et le torse aurait été protégé jusqu'aux reins par ce que Boppert a identifié comme une cotte de mailles (lorica hamata), une protection que l'on ne distingue guère aujourd'hui (Boppert 1998, p. 80). C'est, en effet, une cotte de mailles que porte Togitio (Mayence, milieu du $\mathrm{I}^{\mathrm{er}}$ siècle : Esp. 5870; Boppert 1992, $\left.\mathrm{n}^{\circ} 32\right)$ : même si le relief est particulièrement usé, on y distingue nettement les protections renforcées des épaules et des bras, peut-être encore lisibles sur les bras d'Argiotalus. Malgré l'usure de la pierre, on peut identifier sur notre stèle un baudrier en sautoir sur l'épaule gauche et un mince ceinturon de cuir à boucle en losange, orné et renforcé par deux plaques triangulaires, pointe vers la boucle, de part et d'autre de celle-ci, à moins que la torsade $\mathrm{du}$ bourrelet de la taille, également figurée sur la statue du guerrier de Vachères (Vaucluse, fin du $\mathrm{I}^{\mathrm{er}}$ siècle), ne montre la présence d'un enserrement souple de la taille, et par conséquent la présence, non pas d'un ceinturon décalé, mais d'une sorte de " ceinture de flanelle " analogue à celle du paysan et du militaire de la première moitié du $\mathrm{xx}^{\mathrm{e}}$ siècle, ce qui correspondrait à son évolution générale à partir du $\mathrm{I}^{\mathrm{er}}$ siècle av. J.-C. (communication d'André Rapin). Seul est encore bien lisible le grand bouclier celtique qui protège la jambe, le flanc et l'épaule gauches du cavalier ainsi que le ventre de la monture (Zieling 1989, p. 352, fig. 22) : on en voit la face interne derrière l'encolure du cheval et, à l'extrémité du bras gauche, un léger surcreusement marque peut-être le creux de l'umbo métallique. Il est ovale pour ne pas blesser la monture.

L'armement offensif se borne ici à une lance courte (hasta) ou plutôt une javeline, arme caractéristique du cavalier romain de harcèlement (Flavius Josèphe, BJ, III, V, 5, 27, 96-97; Vigneron 1968, p. 269). Argiotalus ne la brandit pas pour percer un ennemi à terre mais la saisit à mi-hampe, de la main droite relevée en arrière, dans un geste large qui annonce le jet. C'est à peu de choses près la posture, rare, du cavalier C. Tutius, à Mayence, qui porte cependant une épée longue sur la cuisse droite (Esp. 5789). Paradoxalement, Argiotalus, comme Maris, son voisin de la nécropole de Worms, ne porte aucune arme de combat rapproché telle que la longue et large " latte " de cavalerie des Gaulois (spatha), ordinairement portée au flanc droit ${ }^{10}$; pas même une épée courte de type gaulois, à poignée anthropomorphe, arme plus " aristocratique » dont le type celtique est originaire d'Europe centrale, ni même un poignard.

"Quant aux cavaliers, ils portent une grande épée au côté droit, une longue pique à la main, un bouclier long posé en écharpe contre le flanc du cheval, et, dans un carquois, trois dards ou davantage, à large pointe et aussi longs que des javelots. Leurs casques et leurs cuirasses sont les mêmes que ceux des gens de pied. Les cavaliers d'élite qui forment l'escorte du général sont armés comme leurs camarades de la ligne. "Flavius Josèphe, BJ, III, V, 5, 27 (96-97).

L’absence d'épée est relativement rare sur les reliefs funéraires des cavaliers d'ailes (à Bonn, Esp. 6248, par ex.). Les rares stèles de cavaliers ne portant pas la spatha sont celles, plus tardives, de légionnaires et d'archers montés : l'épée longue ne fait pas partie de leur armement offensif ${ }^{11}$.

\section{Caractères généraux du bas-relief}

L'iconographie du cavalier chargeant un ennemi à terre dérive de la stèle grecque de Dexileos (Athènes, musée du Céramique, 394 av. J.-C. : Brilliant 1963, p. 54). De nombreuses stèles rhénanes reproduisent cette charge (stèles de Caius Romanius, de Petronius Disacentus, d'Annauso ou d'Andes). Sans vaincu à terre foulé par le cheval, comme sur les reliefs funéraires de Maris, Togitio ou Flavius Proculus à Mayence, le monument d'Argiotalus ne reproduit qu'une partie du poncif. La présence d'une chabraque, l'allure du cheval, son port de tête et l'étonnante absence de spatha pourraient évoquer un exercice de parade, mais les stèles funéraires qui représentent des chevaux défilant sans cavalier sous la conduite du calo, montrent un long tapis purement décoratif posé sans selle sur le dos du cheval et non la fine selle avec chabraque de la monture d'Argiotalus ${ }^{12}$.

10. À Worms, Esp. 6014, 6016, 6018, 6033, 6035; à Mayence, Esp. 5785, 5786, 5852, 5854, 5870; à Bonn, Esp. 6282, 6283, 6292; à Cologne, Esp. 6435.

11. Stèle d'un cavalier maure, brandissant une javeline comme Argiotalus, à Neapolis (Palestine), fin du $\mathrm{II}^{\mathrm{e}}$ siècle (Junkelmann 1991, p. 74-75, fig. 33), ou stèles de cataphractaires du Iv siècle (ibid., p. 7475, fig. 31 et 34), qui appartiennent à un type de cavalerie lourde, de rupture, éloigné du type de l'aile de cavalerie chargée du harcèlement et de la poursuite à laquelle appartenait notre Gaulois. Sur les armes défensives et offensives, voir Hamdoune 2007 et Mathieu 2007.

12. Stèles d'Oclatius et de Viruno (Junkelmann 1991, fig. 77; Speidel $1994, n^{\circ}$ 2), revue des troupes sur la colonne Aurélienne (Speidel 1994, 
Si aucune trace de polychromie n'a été conservée sur ce relief endommagé par les intempéries, la pollution et la lumière, il faut bien évidemment l'imaginer rehaussé de couleurs vives posées en aplats sur un épais enduit blanc, comme le montre la polychromie restituée d'après les traces relevées sur la stèle du cavalier Silius, de Dienheim, conservée à Mayence (Cüppers 1990, pl. coul. 4). Aussi violente que celle des urnes étrusques ou des santons de Provence, cette polychromie en précisait les détails : du gris ou du jaune pour la cotte de mailles et le casque métalliques, du rouge ou du brun sur la ceinture de cuir, du brun pour la robe du cheval et le bois de la javeline, du rose pour les carnations, du noir ou du brun pour les yeux et les sabots et du rouge pour rendre plus lisible l'inscription.

Cette stèle est d'un schéma bien connu en Germanie mais son interprétation, originale à certains égards, précède la fixation définitive du type ou s'est libérée de certaines contraintes : le geste large est inhabituel, la mise en espace du sujet est de piètre qualité et son intégration dans la conque nie le fronton qui l'abrite. Le traitement " géométrique » du corps du cheval et des membres, grêles, ainsi que le caractère sommaire du décor végétal, incompris sur des acrotères informes, désignent une sculpture peu savante et assez maladroite. Bref, ce n'est pas du grand art! Comme celle de Maris, par exemple, cette stèle présente un relief assez mal proportionné, à l'intégration presque maladroite. Mais la datation précoce de ce monument, qui emprunte pourtant aux traditions funéraires gréco-romaines, en fait un quasi prototype : la stèle d'Argiotalus fait partie des premiers monuments funéraires figurés en Rhénanie, sous le règne de Tibère (Holder 1980, p. 147).

Par son type, par le style de sa sculpture, par la graphie de l'inscription, et même par la taille de la monture, cette stèle doit être datée de la première moitié du $\mathrm{I}^{\mathrm{er}}$ siècle. L'histoire de l'ala Indiana permet de préciser la date de la mort du cavalier et, donc, de l'érection de son monument.

\section{Argiotalus et l'armée romaine}

Argiotalus appartenait à l'ala Indiana, un corps de cavalerie auxiliaire, étranger, dont le nom complet, ala Gallorum Indiana, figure sur un diplôme militaire daté de 134 (CIL XVI, 80 ; Alföldy 1968, p. 19). L'épithète "Gallorum » indique le peuple parmi lequel l'unité a fait son premier recrutement. Seules les unités d'auxiliaires recrutées entre Garonne et Seine sont dites "Gallorum », par référence aux grandes divisions ethniques de la Gallia comata, antérieures

$\left.\mathrm{n}^{\circ} 3\right)$. Un tapis de selle orné et moins long est identifiable sur le cheval de Silius Attonis, mené à la parade par son calo $\left(2^{\mathrm{e}}\right.$ moitié du $\mathrm{I}^{\mathrm{er}}$ siècle : Boppert 1992, n 52). à la réforme administrative d'Auguste entre 27 et 16-13 av. J.-C. (Tassaux 1996, p. 153-154), ce qui exclurait la région de Trêves si cette épithète avait été attribuée précocement, et aurait renforcé l'hypothèse d'un recrutement dans le Val de Loire lors de la première « affaire » de 21. Pour autant, Trêves se situe bien dans l'une des Trois Gaules, la Belgique. Sur l'ensemble du cours du Rhin, à l'époque julio-claudienne, les unités de cavaliers et de fantassins auxiliaires étaient en grande partie originaires de Gaule et de Germanie (Alföldy 1968, p. 136-137).

\section{L'ala Indiana}

Comme le précise Tacite (Ann., III, 42, 1), l'ala Gallorum Indiana a donc été créée à Trêves, en Gaule Belgique, non loin du camp de sa légion de rattachement (Mayence) et dans la cité du chef qui donna son nom à l'unité : l'aile Indiana tient son nom de l'un de ses commandants, sans doute le premier, Iulius Indus, un Trévire mentionné par Tacite pour avoir combattu dans l'armée romaine, à la tête d'une unité de cavalerie recrutée dans son peuple, contre les insurgés du grand soulèvement de la Gaule du nord-est suscité par son compatriote, le Trévire Iulius Florus, et par l'Éduen Iulius Sacrovir. Cette insurrection faisait suite à la révolte, dans l'Ouest, des Andécaves et des Turons, voisins des Namnètes, en 21 (Ann. III, 42 et 46). Iulius Indus fut sans doute récompensé de sa loyauté et de ce fait d'armes par l'octroi de son nom à l'unité, formellement constituée ou non, qu'il commandait déjà (Birley 1978, p. 257 et 267, $\left.\mathrm{n}^{\circ} 13\right)$.

Il n'est pas impossible que l'ala Indiana procède d'un corps franc privé : la troupe était commandée par un chef trévire bénéficiant de la citoyenneté romaine, qui dut «amener avec lui ses clients et ambactes, ses hommes liges " (Tassaux 1996, p. 148-149). Initialement constitué des "vassaux » de son chef, ce corps franc avait peut-être été rassemblé par Iulius Indus de sa propre initiative pour contribuer à réprimer l'insurrection mais, lors du soulèvement de Florus et Sacrovir dans les Ardennes et en Bourgogne, la seconde " affaire " de 21 , ces cavaliers gaulois semblent constituer déjà une unité régulière de l'armée romaine ${ }^{13}:$ Tacite précise que cette

13. Cf. Saddington 1970, p. 99 et p. 100-101; 1975, p. 182. Très vague, l'expression cum delecta manu ne précise pas la taille de la troupe engagée mais, chez Tacite, s'applique à un détachement auxiliaire. Le terme delectus désigne le plus souvent des forces spéciales, tandis que Tacite désigne aussi par manus des recrues indigènes utilisant leurs propres méthodes, comme le contingent de Maurétaniens employé par les Romains lors de la révolte de Tacfarinas en 24 apr. J.-C. (Ann., IV, 24, 3). Même si l'unité semble effectivement constituée principalement de Trévires (" e Treviris", dans Ann., III, 42, 1), ce n'est pas dans ce sens qu'il faut comprendre le passage ultérieur du texte (Ann., III, 46, 2), comme le fait par erreur Saddington (1970, p. 99 et 103.1975 , p. 182) : il ne faut pas lire ala Trevirum (« une 
troupe de combat avait la discipline et les modes de combat des Romains ("militia disciplinaque nostra habebatur", Ann., III, 42, 1). En outre, cette unité ne manifeste déjà plus d'homogénéité ethnique : elle comprend des cavaliers trévires, mais aussi, pour autant qu'on le sache par les épitaphes antérieures à 43 , date du départ de sa légion de rattachement accompagnée de ses corps d'auxiliaires pour la conquête de la Britannia, elle comprend alors aussi au moins un Namnète (estuaire de la Loire) et un Ubien (région de Cologne), plus sans doute des cavaliers d'autres civitates gauloises, car l'ala Indiana est l'une de ces unités dont le recrutement est mixte dès la fin de l'époque augustéenne (Alföldy 1968, p. 19).

Au sein de troupes indigènes que l'on peut comparer aux goums de l'armée française durant la seconde guerre mondiale (Louis Maurin), les ailes de cavalerie représentent une élite relative (" une troupe d'élite ", écrit Tacite à propos de l'ala Indiana). L'organisation militaire, telle qu'on la connaît pour le cours inférieur du Rhin et dans d'autres provinces (Alföldy 1968, p. 141), devait compter presque autant d'auxiliaires (" étrangers ") que de légionnaires (20000 auxiliaires environ pour 22000 légionnaires) avec, parmi les auxiliaires, un cavalier pour trois ou quatre fantassins. L'unité d'Argiotalus est une aile quingénaire (à 500 hommes), avec 16 turmes de trois décuries, soit 480 hommes commandés par un préfet appartenant à l'ordre équestre et par un sous-préfet, officiers assistés d'un décurion princeps et de 16 décurions (Le Bohec 1989, p. 26-27; Junkelmann 1991 , p. 59 et 62 ; Dixon et Southern 1992, p. 23). Ses missions étaient la reconnaissance en territoire ennemi, le harcèlement, la riposte rapide, les escarmouches mais aussi le maintien de l'ordre et la police des populations indigènes.

Sans doute très peu de temps après la création de l'aile Indiana, l'un de ses cavaliers, Argiotalus, a été recruté en Gaule Lyonnaise, dans la cité des Namnètes : cette aile de cavaliers trévires a eu l'opportunité de compléter sa troupe en enrôlant un Namnète et sans doute d'autres gens de l'Ouest au printemps ou au début de l'été 21 , lors de l'opération en Val de Loire de la legio XIIII Gemina, dont elle dépendait, pour mater la rébellion des Andécaves et des Turons avant de revenir " traiter ", dans les Ardennes, celle du Trévire Iulius Florus et de l'Éduen Iulius Sacrovir (Ann. III, 42 et 46; Birley 1978, p. 257, et 267, n. 13).

\section{L'ala Indiana et les révoltes de 21}

Tacite raconte comment le Trévire révolté Iulius Florus tente d'entraîner dans sa rébellion une aile de cavalerie levée à Trêves - donc principalement composée de ses compatrio-

aile de Trévires "), mais una ala Trevirum [...] profligavere : " une seule aile de cavalerie [suffit] à battre le Trévire ", sous entendu Iulius Florus. tes - pour la retourner contre Rome; certains cavaliers se laissent corrompre mais la majorité des recrues reste fidèles à Rome.

"Sur ces entrefaites, Florus poursuivait ses projets et tâchant de gagner à sa cause une aile de cavalerie qui, enrôlée parmi les Trévires, était exercée à la romaine et soumise à notre discipline, l'engageait à commencer la guerre par le meurtre des trafiquants romains. Quelques cavaliers seulement se laissèrent corrompre. Les plus nombreux demeurèrent dans le devoir. Mais la foule des débiteurs et des clients de Florus prit les armes et tous se dirigeaient déjà vers les défilés boisés que l'on nomme Ardenne, quand ils furent repoussés par les légions de l'une et l'autre armées que Visellius et $C$. Silius avaient lancées à leur rencontre par des chemins opposés. On envoya aussi en avant-garde, avec une troupe d'élite, Iulius Indus, de la même cité que Florus, mais qui ne partageait pas ses idées et était par conséquent plus ardent à faire bonne besogne. Il avait affaire à une multitude encore en désordre : il la dispersa. Florus, à la faveur de retraites mal connues, trompa d'abord ses vainqueurs; mais enfin, à la vue des soldats qui occupaient les issues, il se suicida. Telle fut la fin de la révolte des Trévires. " (Ann., III, 42, 1-4.)

Commandées par Visellius Varro et C. Silius Caecina, commandant de l'armée du Rhin supérieur de 14 à 21 (Franke 2000, p. 192), les légions XIV Gemina et XVI ${ }^{e}$ Gallica marchent contre Florus mais c'est surtout Iulius Indus, son compatriote "qui ne partageait pas ses idées" qui, "envoyé en avant-garde avec une troupe d'élite ", disperse les révoltés et provoque la fuite puis le suicide de Florus, chef des rebelles. À quoi Tacite fait-il référence lorsqu'il écrit "cum delecta manu », c'est-à-dire " avec une troupe d'élite »? S'agit-il de l'ala Indiana entière ou seulement d'un détachement de cette unité, ou encore d'un corps constitué de détachements (vexillations) de plusieurs unités différentes? Au cours de son récit, l'historien revient sur ces événements et confirme qu'il s'agissait bien d'une aile seulement : "una ala Trevirum [...] profligavere" : "une aile de cavalerie [a suffi] à vaincre le Trévire » (Ann., III, 46, 3). En tout état de cause, cette troupe de cavaliers restés loyaux, au rôle décisif, a pu être récompensée par le légat C. Silius Caecina, ou par son successeur Sulpicius Galba en étant constituée en unité régulière si elle ne l'était déjà, ou plutôt en se voyant attribuer le nom de son glorieux commandant si l'unité était bien déjà constituée. L'attribution à l'aile de son nom permanent, sinon sa création, est donc sans doute immédiatement consécutive aux événements de 21 (ILS 2496; Alföldy 1968, p. 19; Birley 1978, p. 267, n 13, et p. 272273; Holder 1980, p. 21).

Iulius Indus et les chefs insurgés étaient du même monde, et sans doute tous trois officiers dans l'armée romaine. Leur gentilice "Iulius» indique que leurs familles bénéficiaient de la citoyenneté romaine depuis quatre générations au plus et 
avaient obtenu le droit de cité pour bons et loyaux services envers César, plutôt qu'Auguste. Aristocrates l'un trévire et l'autre éduen, Florus et Sacrovir utilisèrent le mécontentement provoqué par la pression des negociatores et la levée des tributs pour trahir, se rebeller et soulever leurs compatriotes contre les Romains. Tacite montre l'importance de cette insurrection : plusieurs cités gauloises se révoltèrent, des commerçants romains furent massacrés et le chef-lieu de la cité des Éduens, "peuple frère " et fidèle allié de Rome, Autun, l'une des rares villes précocement remparées de la Gallia comata et protégée d'une puissante muraille augustéenne de $6 \mathrm{~km}$ de long, fut même prise et pillée par les insurgés. Cette révolte frappa les imaginations et suscita l'envoi des légions depuis Mogontiacum-Mayence. Elle était d'autant plus dangereuse pour Rome que les Trévires et les Nerviens, peuples gaulois de la rive gauche du Rhin, étaient parents des tribus germaines de la rive droite restées indépendantes. En outre, pour avoir servi comme officiers de l'armée romaine, ces chefs gaulois étaient formés à ses méthodes de combat et les retournaient contre Rome lorsqu'ils se révoltaient. Ils représentaient un risque d'autant plus grand.

Les Namnètes semblent s'être définitivement soumis à Rome après la défaite des Vénètes en 56 : César ne les cite pas au nombre des peuples qui contribuent à l'armée de secours à Alésia et Tacite les ignore lorsqu'il évoque la révolte de leurs voisins Andécaves et Turons en 21. Argiotalus pourrait donc avoir été recruté peu avant l'intervention contre Florus et Sacrovir par la legio XIIII Gemina (ou seulement un détachement de cette légion?) lancée contre les Andécaves et les Turons pour mater, dans l'Ouest, une révolte qui s'est développée peu après dans l'Est. Il est donc possible qu'Argiotalus ait fait partie d'un contingent de cavaliers fourni à l'armée romaine à titre de tribut par les civitates armoricaines placées "dans la catégorie la moins favorisée, celle des cités sujettes (ou stipendiaires)" (Galliou 1983, p. 41).

\section{Les garnisons de l'ala Indiana}

Sous Tibère, l'aile "gauloise » d'Argiotalus est stationnée sur la frontière nord de l'Empire, une région qu'Auguste s'est employé à sécuriser face aux Germains après l'échec de la tentative de constitution d'une Germania Magna par la conquête de la Germanie jusqu'à l'Elbe (15-13 av. J.-C.) et le désastre de Varus (9 av. J.-C.). L'armée romaine avait alors pour mission de contrôler le Rhin, à la fois frontière et voie de communication. L'aile Indiana appartenait ainsi à l'organisation défensive de l'Empire contre les Germains, fondée sur les puissants camps légionnaires de Mayence et sur des "points d'appui » secondaires qui, comme Worms, jalonnaient la rive occidentale du Rhin. Ces petites garnisons étaient constituées de plusieurs corps d'auxiliaires associés, voire de détachements de différentes unités et de vexillations légionnaires. Stationnée à Worms, l'aile Indiana dépendait de la legio XIIII Gemina et aurait donc quitté avec elle les rives du Rhin pour Cirencester lorsque cette légion y fut engagée par Claude à partir de 43 pour la conquête de la Bretagne insulaire ${ }^{14}$. La présence de sa stèle à Worms, en Germanie, lieu de garnison précoce de l'ala Indiana, montre qu'Argiotalus a disparu avant le transfert de son unité en Britannia, en 43.

Outre Argiotalus, on connaît plusieurs cavaliers de l'aile Indiana (Spaul 1994, p. 152-153, n ${ }^{\circ}$ 52, Ala Gallorum Indiana), dont les dates sont malheureusement imprécises. - À Mayence-Weisenau, stèle funéraire du cavalier ubien Fronto, datée de la $1^{\text {re }}$ moitié du $\mathrm{I}^{\text {er }}$ siècle (Selzer 1988, $\mathrm{n}^{\circ} 76$ ).

- À Mayence, sarcophage de Sequentia Faustina, épouse de Fl. Flavianus Aventinus, décurion de l'aile Indiana (CIL XIII, 7028).

- À Klein-Winternheim (près de Mayence), sarcophage de Marcellinia Marcella, épouse de Iulius Paterninus, décurion de l'aile Indiana (CIL XIII, 7257).

- À Cologne, Albanius Vitalis, cavalier trévire de l'aile Indiana, de la turme de Barbus, ayant probablement servi entre 80 et 100 en Germanie Inférieure, est représenté héroïsé, drapé à la romaine et à demi étendu sur le lit de son banquet funèbre, avec table aux victuailles et serviteur, tandis qu'un second registre montre son valet cuirassé et armé d'un faisceau de javelines, conduisant par la bride son cheval richement caparaçonné (CIL XIII, 8519; Esp. 6460; Alföldy 1968, p. 20 et $\mathrm{n}^{\circ} 31$ ); Alföldy estime que Albanius Vitalis possédait le droit latin.

- À Neckarburken, diptyque en bronze, diplôme de Trajan pour les cavaliers et fantassins des ailes I (dont l'ala I Indiana Gallorum) et des cohortes XV (CIL XIII, 6495).

Les quatre soldats dont les monuments ont été trouvés à côté de celui d'Argiotalus appartenaient à des unités de fantassins ou de cavaliers différentes de la sienne, ce qui montre

14. Au moins une aile et deux cohortes auxiliaires étaient attachées à chaque légion (Alföldy 1968, p. 137). Hassal (2000, p. 442, n. 5) estime que l'aile Indiana était bien stationnée à Cirencester dès la Conquête, c'està-dire à partir de 43. Certains auteurs considèrent qu'il n'est pas impossible que ce transfert n'ait eu lieu qu'en 60-61, lorsque Néron dut faire face à la révolte de Boudica, reine des Icènes, dans la région de Norwich (Norfolk). Sur l'ala Gallorum Indiana, cf. Alföldy 1968, p. 19; Holder 1980, p. 21 ; 1982 , p. 15, 106 et 108, qui optent pour le transfert de l'unité en Bretagne sous Néron, en 60 ou 61, lors de la révolte de Boudica (Cichorius 1893, col. 1243-1244; Le Bohec 1990, p. 178-179 et 218-219, d'après Ritterling 1925). Il faut toutefois corriger la liste et la chronologie de ces différentes garnisons : l'aile a d'abord stationné à Worms, en Germanie supérieure, comme l'atteste la stèle d'Argiotalus, puis en Germanie Inférieure à son retour de Bretagne, avant de regagner Mogontiacum - Mayence entre 117 et 134, alors que Cichorius (1900, col. 1243-1244) la situe d'abord en Germanie Inférieure, puis en Germanie Supérieure. Elle reçoit sous Trajan le surnom de Pia Fidelis, durant la guerre Batave. 
la multiplicité des détachements présents simultanément à Worms. La présence à Mayence-Weisenau et à Cologne, en Germanie Inférieure, entre 80 et 100 , donc après le départ de leur unité en Bretagne, de deux cavaliers de l'aile Indiana, l'Ubien Fronto, de la région de Cologne (Selzer 76, $1^{\text {re }}$ moitié du $\mathrm{I}^{\text {er }}$ siècle) et le Trévire Albanius Vitalis, montre qu’à certaines périodes, l'aile était partagée en plusieurs cantonnements et il n'est donc pas impossible que seul un détachement de ce corps ait pu stationner à Worms, un autre à Cologne (?), le reste de l'unité étant demeuré à Mayence, à proximité de sa légion de rattachement, ou, plus tard, l'accompagnant en Britannia.

$A u$ I $^{\text {er }}$ siècle, la cavalerie auxiliaire était principalement recrutée dans les régions celtisées, en Belgique, en Lyonnaise et en Germanie notamment. Les unités devaient être régulièrement complétées de recrues « indigènes » sélectionnées au gré des garnisons successives, malgré l'ethnique d'origine de l'unité. Rien d'étonnant, donc, à la présence d'un Namnète dans les rangs de l'aile Indiana.

\section{Le cavalier Argiotalus}

L'iconographie de la stèle et l'armement d'Argiotalus confirment l'épitaphe : l'homme au bouclier ovale et à la javeline caractéristiques appartient bien à une unité auxiliaire de cavalerie formée de supplétifs de cultures diverses, mais barbares et issus de régions conquises par Rome. Dans la grande majorité des cas, surtout au début de l'Empire, sous les Julio-Claudiens, les auxilia, cohortes d'infanterie ou ailes de cavalerie comme l'aile Indiana, sont des unités de pérégrins. Leurs soldats obtiennent habituellement la citoyenneté romaine au terme de leur carrière militaire, après quelque vingt-cinq années de service s'ils ont la chance d'être encore en vie, mais l'attribution de la citoyenneté romaine à la fin du service n'est réellement attestée, par des diplômes militaires, qu'à partir du règne de Claude, donc un peu après l'époque d'Argiotalus (Junkelmann 1991, p. 63-65).

Argiotalus ne devint jamais citoyen romain puisqu'il mourut après dix ans de service militaire seulement : il en fallait alors environ vingt-cinq pour être rendu à la vie civile et récompensé du droit de cité. S'il s'était enrôlé - ou l'avait été d'autorité au titre de la livraison de soldats par les cités stipendiaires - peu avant ou peu après 21 , il mourut au plus tard entre 31 et 43 , date du départ de son unité pour la conquête de la Britannia. La coutume étant d'arrondir l'âge du défunt à un chiffre se terminant par 0 ou par 5 , à sa mort, Argiotalus avait à peu près 30 ans. Il aurait donc été enrôlé à l'âge d'environ 20 ans, entre 18 et 21 ans comme le voulait la pratique (Le Bohec 1990, p. 65-66 et 76). Il serait donc né en Armorique entre 1 et 13 , à la fin du règne d'Auguste.
Les soldats pouvaient financer leur propre monument funéraire en économisant sur leur solde. Les deux dernières lignes de l'épitaphe d'Argiotalus précisent que son monument a été commandé et payé par ses héritiers, c'est-à-dire soit par ses enfants et leur mère - même s'ils ne pouvaient légalement se marier, beaucoup de militaires vivaient en concubinage $^{15}$ - soit plus probablement par ses compagnons d'armes, qui se seraient cotisés pour ériger sa stèle comme c'était fréquemment le cas. Voisin de nécropole d'Argiotalus, le Trévire Partus a été incinéré à la charge de ses frères - ils étaient donc au moins deux - qui avaient aussi payé l'érection de son monument : c'est donc toute la fratrie de Partus qui semble s'être enrôlée en même temps, sans doute dans la même unité.

\section{Les événements de 21 et la datation de la stèle d'Argiotalus}

Les critères typologiques, stylistiques et archéozoologiques ainsi que le contexte historique et militaire permettent de situer l'existence d'Argiotalus durant la première moitié du $\mathrm{I}^{\mathrm{er}}$ siècle, et même avant le règne de Claude. L'oblique presque rectiligne tracée par la queue, la croupe, le dos et la tête du cheval est comparable à celle que l'on observe sur le relief tibérien du cavalier Maris. Enfin, le volume allongé et cylindrique du cheval et la petite taille caractéristique des montures gauloises antérieures au milieu du $\mathrm{I}^{\text {er }}$ siècle sont semblables à ceux des stèles de Cantaber et de Rufus, datées de la fin du règne de Tibère, dont la selle, les rênes et la cotte de mailles sont similaires à celles d'Argiotalus (Mayence, Esp. 5784; Boppert 1992, n 30). On peut raisonnablement conjecturer que ce cavalier servit dans l'armée romaine dès les premiers temps de l'aile Indiana, sous le règne de Tibère, sans doute peu avant ou à l'occasion des événements de 21 qui peuvent justifier la constitution de l'aile ou l'honneur fait à son chef, d'une part, et le recrutement d'un Gaulois dans l'Ouest de la Lyonnaise, d'autre part.

L'origine d'Argiotalus est cohérente avec l'épithète Gallorum officiellement conférée à l'ala Indiana et sa carrière peut sans doute être mise en relation avec l'origine de l'unité : au vu de la datation haute proposée, antérieure au règne de Claude, il est assez probable que le Namnète a servi sous les ordres du Trévire Iulius Indus lui-même, premier

15. Il s'agit évidemment d'une tolérance : " toute union [des militaires] est prohibée d'Auguste à Claude, tolérée de Claude à Septime-Sévère » et il faudra attendre Septime-Sévère (146-211) pour que le concubinage soit autorisé. Le mariage officiel n'est autorisé qu'à la fin d'un service militaire d'environ vingt-cinq ans (Le Bohec 1989, p. 244). Les fouilles de camps, en Angleterre, ont montré qu'avant cette reconnaissance légale, les soldats vivaient parfois en concubinage et avec leur famille : des objets " civils " (une chaussure d'enfant, notamment, d'après Michel Reddé!) ont été trouvés à l'intérieur même des camps, montrant que des soldats vivaient bien maritalement. 
préfet de l'aile, et qu'il a participé à la répression des révoltes de 21. Le Val de Loire avait précédé les Trévires et les Éduens dans le soulèvement gaulois : "Il n'y eut presque pas de cités où ne fussent semés les germes de cette révolte, mais ce furent les Andécaves [voisins des Namnètes] et les Turons [voisins des Andécaves] qui éclatèrent les premiers. Les Andécaves furent réduits par le légat Lucilius Aviola qui fit marcher une cohorte tenant garnison à Lyon. Les Turons furent défaits par un corps de légionnaires que le même Aviola reçut de Visellius Varro, gouverneur de Germanie inférieure et auxquels se joignirent des nobles gaulois. " (Tacite, Ann., III, 41, 1-3) ${ }^{16}$.

Comme le souligne Tacite, à l'instar du Trévire Iulius Indus peu après cette révolte, des aristocrates gaulois de l'Ouest de la Lyonnaise, et peut-être des Namnètes (mais Argiotalus n'est qu'un simple cavalier), ont également combattu aux côtés de l'armée romaine pour mater la révolte des Andécaves et des Turons, prolongée dans l'Est par celle de Iulius Florus et de Iulius Sacrovir.

\section{Des camps témoignant de la révolte de 21 dans l'Ouest?}

On ne connaissait jusqu'ici aucun témoignage archéologique du soulèvement de certaines cités de l'Ouest en 21 mais, sous réserve du contrôle archéologique indispensable, il est possible que l'archéologue aérien Gilles Leroux (INRAP) ait fait, en 2006, au moins deux découvertes majeures à ce sujet : au lieu-dit Les Vordeaux, dans la périphérie de Cholet (Maine-et-Loire), il a photographié une vaste enceinte fortifiée rectangulaire d'environ deux hectares, à fossés palissadés, dotée de deux tours d'angle circulaires protégées de fossés ou de douves (?), d'une entrée unique à titulum interne et de traces d'agrandissements progressifs (fig. 3). Une enceinte similaire, très régulière, a également été repérée au sud de la Loire, à La Chapelle-Heulin (Loire-Atlantique), au lieudit le Moulin-des-Landes (fig. 4), et d'autres encore dans le Morbihan, l'Ille-et-Vilaine et le Maine-et-Loire voisins (comm. pers.). Ces sites évoquent l'organisation de camps d'aile ou de cohorte (celle de Lyon?). Découvertes par archéologie aérienne, ces anomalies végétales n’ont pour l'instant fait l'objet d'aucune vérification au sol et ne sont pas datées. Malgré une typologie inhabituelle, elles pourraient encore faire partie de ces myriades d'enclos laténiens

16. La cohorte de Lyon ne put suffire à écraser les Andes et les Turons et il fallut faire donner des troupes expédiées de Germanie; les inscriptions CIL XIII, 1121, 1122, 1123, trouvées à proximité du camp de vexillations d'Aulnay-de-Saintonge (Charente-Maritime), dont l'implantation semble précisément liée à la révolte de 21 , mentionnent deux légions alors stationnées à Mayence : la legio II Augusta et la legio XIIII Gemina, celle-là même à laquelle était ou fut rattachée l'ala Indiana Gallorum d'Argiotalus (Tronche, 2006, p. 207).

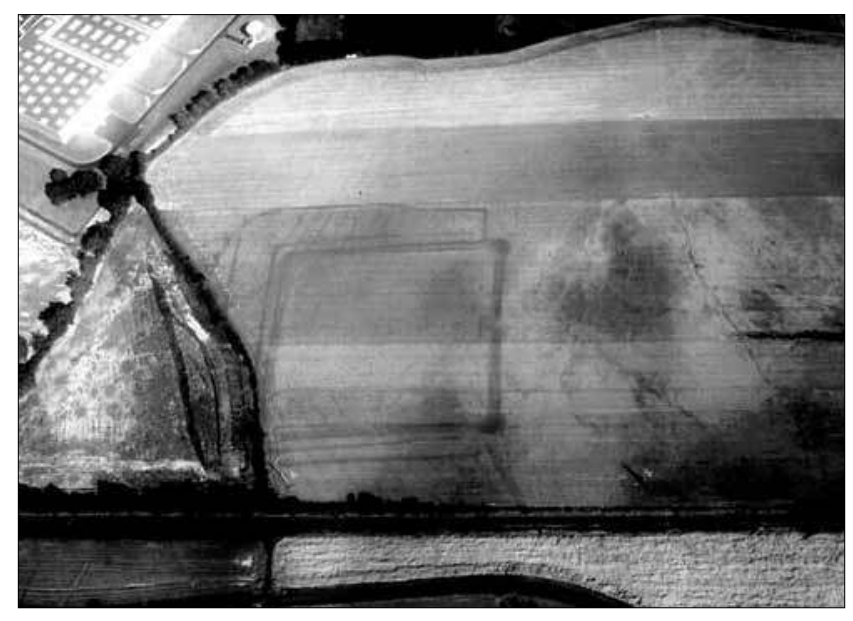

Figure 3 : Enceinte fortifiée des Vordeaux, à Cholet (Maine-etLoire), prospection aérienne de Gilles Leroux, 18 juin 2006 : ferme gauloise ou, plutôt, camp romain de cohorte ou d'aile? (Cl. Gilles Leroux, INRAP Grand Ouest).

Figure 3: Les Vordeaux fortified enclosure, Cholet (Maine-et-Loire), aerial photograph by Gilles Leroux on 18th june 2006: a Gaulish farmstead, or rather a Roman camp for a Cohort or a Wing?

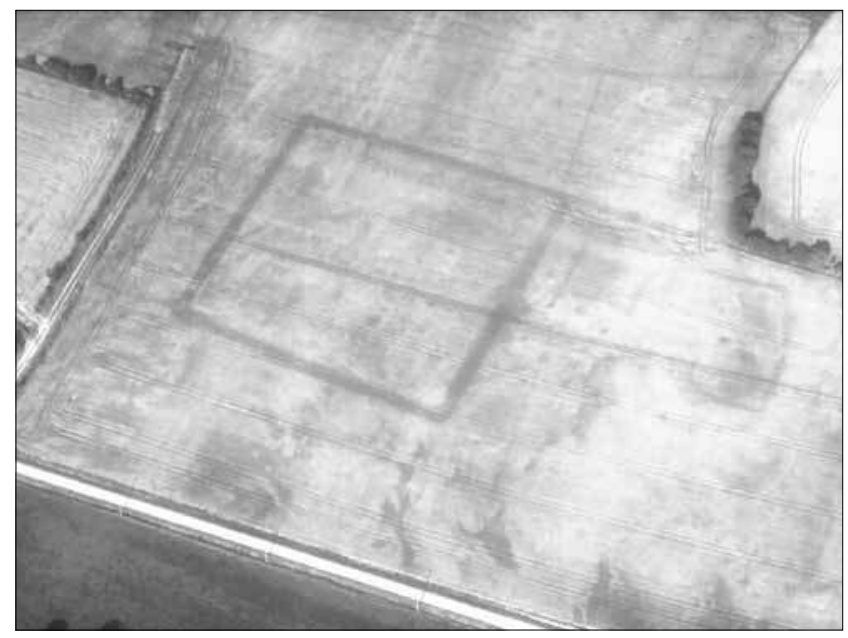

Figure 4 : Enceinte fortifiée du Moulin-des-Landes, à La ChapelleHeulin (Loire-Atlantique), prospection aérienne de Gilles Leroux, 2006 : ferme gauloise ou, plutôt, camp romain de cohorte ou d'aile? (Cl. Gilles Leroux, Inrap Grand Ouest).

Figure 4: Moulin-des-Landes fortified enclosure, La Chapelle-Heulin (Loire-Atlantique), aerial photograph by Gilles Leroux in 2006: a Gaulish farmstead, or rather a Roman camp for a Cohort or a Wing?

déjà identifiés, mais si l'attribution à l'armée romaine de ces installations était prochainement confirmée par la prospection au sol, des sondages ou une fouille, ces sites pourraient concerner soit l'hivernage de troupes de Crassus, de L. Titurius Sabinus ou de D. Brutus lors de la Conquête en 57-56, soit plutôt le cantonnement de troupes d'Aviola 
ou de celles dépêchées de Germanie Inférieure par Visellius Varro lors de la révolte de 21 (détachements des XIV et $\mathrm{XVI}^{\mathrm{e}}$ légions de Mayence), soit encore celui d'une garnison de maintien de l'ordre ou de construction de voies stratégiques peu après cette révolte. Les fouilles du camp d'Aulnay-deSaintonge (Charente-Maritime) - une garnison justifiée par les révoltes de 21 (Tronche 2006) - ont montré que, sous Tibère, la Gaule n'était pas "inermis " comme le prétendait Tacite sans doute par esprit de propagande (Tassaux 1984, p. 149-150, n. 73).

\section{WORMS ANTIQUE ET L'ARMÉE ROMAINE}

\section{La naissance de l'agglomération antique de Worms}

Pour autant que l'on sache et malgré des recherches attentives, aucune agglomération préromaine n'a été trouvée à Worms : la région n'est plus habitée à partir de $70 \mathrm{av}$. J.-C. car les intrusions germaniques sur la rive occidentale du Rhin y interdisent toute installation pérenne. Celles-ci provoquent finalement l'intervention de César contre Arioviste dans cette région. Le Rheinhessen, c'est-à-dire la région de Worms, à l'ouest du Rhin, n'a été réellement occupé par les Romains que sous Tibère, vers 20-30 apr. J.-C. et semble avoir été peuplé de populations loyales à Rome, installées là par les Romains (Grünewald 2006, p. 15-17). On n’y connaît pas d'agglomération civile contemporaine d'Argiotalus (fig. 5) mais il existe quelques indices ténus d'une présence romaine précoce sur le site de Worms, à la fin du règne d'Auguste et sous Tibère : ce sont, avec les stèles de militaires, quelques tessons de céramique sigillée italique, estampillés Ateius, Macca, MPV, Camus, Attius, etc. (Grünewald, 1990b, p. 673), qui ont été trouvés sur toute l'emprise actuelle de la ville, toujours sur la première terrasse du lit majeur du fleuve, une zone non inondable où une agglomération pouvait effectivement s'établir. Cette céramique " fine " à la couleur rouge vif et au brillant jusqu'ici inégalé dans ces régions est sans doute arrivée d'Italie pour les militaires, que ce soit dans leurs propres bagages, dans le train des équipages ou par des marchands.

\section{Le nom de Worms et la reconnaissance administrative de Borbetomagus}

Le toponyme Borbetomagus apparaît pour la première fois dans la Géographie de Ptolémée (II, 9, 9), donc dans la première moitié du II ${ }^{\mathrm{e}}$ siècle de notre ère. Dans l'Itinéraire d'Antonin, entre 355 et 374, on lit Borbitomago, et Borbetomagi dans la Table de Peutinger (dont la copie a précisément été retrouvée à Worms au Xv siècle!) Ce n'est que dans la Notitia Galliarum (VII, 5) que l'on trouve mentionnée la civitas Vangionum, chef-lieu de la cité des Vangions. Le nom correct de l'agglomération semble être celui indiqué sur une borne milliaire de Tongres: [Borb]itomag(us) mais rien ne permet de préciser la date d'apparition de l'agglomération ni de son toponyme (Desjardins 1876-1893, IV, pl. VI; Ihm 1897, col. 719-720).

\section{L'armée romaine à Worms}

Sauf nouvelles découvertes, il faut considérer aujourd'hui que les militaires romains ont probablement bâti un ou plusieurs camps de troupes auxiliaires en rase campagne, sur un site favorable en bordure du Rhin, le fleuve étant alors, à la fois une frontière ${ }^{17}$, une voie de communication et un élément défensif pour ce camp. À cette époque il n'existait pas encore de civitas Vangionum. Depuis le désastre de Varus (9 apr. J.-C.), tout le territoire situé à l'ouest du Rhin (rive gauche), était placé sous l'autorité du commandement militaire de Mogontiacum-Mayence ${ }^{18}$, siège de deux légions, la $X I V^{\hbar}$ Gemina et la $X V I^{a 19}$. Comme les vexillations des légions auxquelles elles étaient rattachées, les unités auxiliaires étaient réparties en diverses garnisons. Dès l'époque augustéenne, le légat de Mogontiacum-Mayence avait placé à Worms un fort puis un ou plusieurs camps de troupes auxiliaires comme le montrent les stèles funéraires de soldats qui y ont été trouvées. Jusqu’à la construction du limes, la rive gauche du Rhin reste du ressort du commandement militaire de Mayence; un gouverneur civil n'est apparu qu'ultérieurement.

17. Drusus et Tibère avaient conduit des expéditions en 10-9 et en 9-7 av. J.-C. en Germanie, dans le but de faire une province romaine du territoire d'entre Rhin et Elbe. En 6 apr. J.-C., le légat C. Sentius Saturninus mène une expédition contre Marbod mais les opérations doivent être arrêtées à cause de soulèvements en Pannonie et en Illyrie. Le désastre de Varus, en 9, met un terme au projet d'Auguste d'établir une province de Germanie allant jusqu'à l'Elbe, et le Rhin est conservé comme frontière de l'Empire (Selzer 1988, p. 31-32).

18. Le légat-gouverneur disposait à Mayence de quatre légions, dont seules des vexillations devaient réellement trouver garnison dans les alentours de la ville tandis que d'autres étaient distribuées sur l'ensemble du territoire. En 43, cette garnison fut réduite à deux légions, la $I V^{h}$ Macedonica et la XXII Primigenia (la seule qui restât à Mayence à partir de 92 apr. J.-C.), par le départ de deux d'entre elles, l'une pour la conquête de la Bretagne, l'autre pour stationner à Novaesium-Neuss (Selzer 1988, p. 30-32).

19. Il s'agit de la legio $X V I^{a}$ Gallica, mais son surnom de Gallica ne semble apparaître dans les inscriptions qu'à l'époque de Claude, alors que la légion est désormais stationnée depuis 43 sur le Rhin inférieur, à la place de la $X X^{a}$ partie en Britannia (Holder 1982, p. 15 et p. 104). La $X X^{a}$ Valeria Victrix est en Germanie Inférieure entre 9 et 43; elle est attestée à Mongotiacum - Mayence (Decker et Selzer 1976, p. 467), puis à Novaesium, sur le Rhin inférieur (Franke 1936, p. 70-71; Selzer 1988, p. 70-71). 


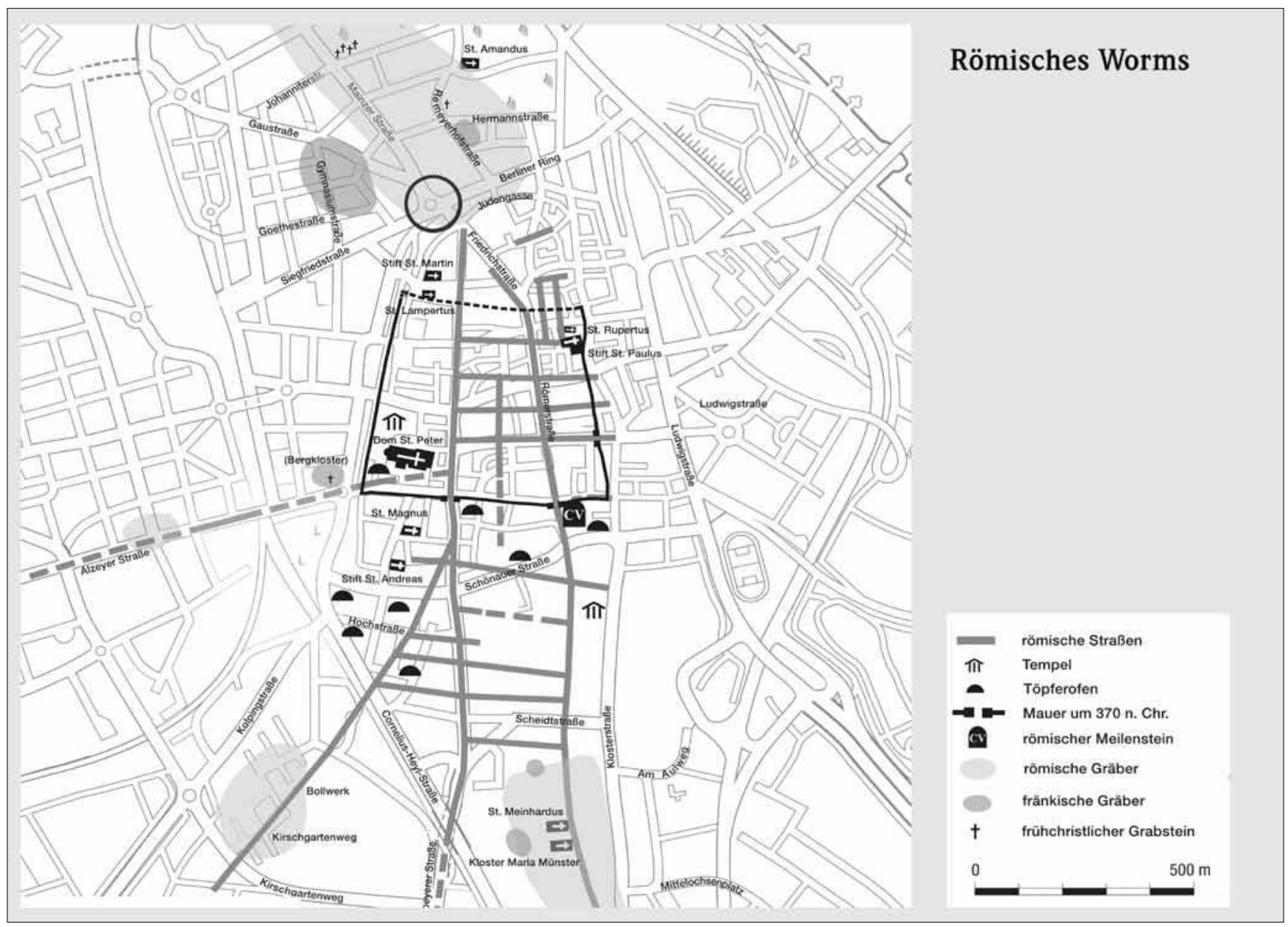

Figure 5 : Worms romaine. Site de découverte de la stèle d'Argiotalus (cercle) : 1, voies ou rues romaines; 2 , temple; 3, fours de potier; 4, rempart de 370 apr. J.-C.; 5, borne milliaire; 6, nécropoles romaines; 7, nécropoles franques; 8 , tombes paléochrétiennes.

Figure 5: Roman Worms, discovery site of the Argiotalus stela: 1, Roman roads or streets; 2, temple; 3, potter's kiln; 4, 370 AD rempart; 5, milestone; 6, Roman cemetery; 7, Frankish cemetery; 8, Paleochristian graves.

Le commandement militaire de la zone de MogontiacumMayence est exceptionnel, vu le nombre de légions stationnées en Germanie : il s'agit d'un commandement supérieur exercé sur la circonscription administrative de l'armée du Rhin supérieur (exercitus superior), ayant son siège à Mayence, et sur celle de l'armée du Rhin inférieur (exercitus inferior), ayant son siège à Cologne (Decker et Selzer 1976, p. 465-466; Fischer 1999, p. 59 et 68). Après 89-90, date à laquelle les deux provinces de Germanie sont réellement créées, le gouverneur de la province de Germanie supérieure siège naturellement à Mogontiacum-Mayence; c'est un gouverneur issu du Sénat, un legatus Augusti pro praetore.

Durant l'époque romaine, quatre ailes de cavalerie et quatre cohortes d'infanterie ont été partiellement ou totalement stationnées à Worms (Bernhard 1990, p. 88) ${ }^{20}$.

20. Sans qu'aucun camp de légion n'ait jamais été établi à Worms, on y a pourtant trouvé l'épitaphe d'un custos armorum de la legio II ${ }^{a}$ Parthica
- L'ala Indiana était l'unité dans laquelle servait Argiotalus (Holder 1980, nº 381 et p. 220).

- L'ala Agrippiana = ala II Flavia Agrippiana, ainsi nommée en l'honneur d'Agrippa, le gendre d'Auguste. Son nom complet n'est connu que par le cursus honorum CIG 3497 (époque d'Antonin le Pieux?). C'est en tout cas la même aile que l'ala Agrippiana mentionnée sur une stèle funéraire de Worms, datée de la période pré-claudienne (CIL XXIII, 6235). De cette époque daterait également l'inscription d'un sous-préfet de l'aile, originaire de Gaule Narbonnaise (CIL XII, 2231; Cichorius 1893, col. 1229). L'aile est recensée en Bretagne par un diplôme de 122, mais peut-être y est-elle arrivée avant la période flavienne (Holder 1980, n 331 et p. 220; Holder 1982, p. 108).

et des briques estampillées de la legio XXII Primigenia (Cüppers 1990, p. 673). Certaines vexillations de légions devaient être en garnison à Worms et certains soldats ont dû y trouver leur dernière demeure... 
- L'ala Sebosiana = ala II Gallorum Sebosiana est l'aile II des Sébosiens ou Ségusiaves, peuple de la Gaule Lyonnaise, région de Feurs (Loire). L'inscription funéraire d'un cavalier de Worms témoigne de sa présence en Germanie au i ${ }^{\text {er }}$ siècle (CIL XIII, $11709=$ CIRh 894). Tacite (Histoires, III, 5) la mentionne dans l'armée de Vitellius, en compagnie de trois cohortes, lors des événements de 69. On a des traces de l'unité en Bretagne insulaire au $\mathrm{II}^{\mathrm{e}}$ siècle, où elle a peut-être participé aux campagnes d'Agricola. Elle est ensuite attestée dans la même province en 103 par le diplôme XXXII (XXI), ainsi que par d'autres inscriptions jusqu'au III $^{\mathrm{e}}$ siècle. Au vu de son surnom de Gallorum, ses premiers membres auraient été recrutés en Gallia comata (Holder 1980, nº 442 et p. 221 ; Holder 1982, p. 109; Cichorius 1893, col. 1246).

- L'ala I Hispanorum ${ }^{21}$ : l'aile I des Espagnols est recensée à Mayence à l'époque julio-claudienne (Holder 1980, n 494, 496 et 497. Selzer 1988, p. 71). Le musée municipal de Worms conserve la stèle funéraire de Carminius Ingenuus, signifer de l'Ala Hispanorum, probablement l'Ala I Hispanorum. Elle daterait du milieu du $\mathrm{I}^{\mathrm{er}}$ siècle (Holder 1980, n 495; Junkelmann 1991, p. 138-139, n 79). Laile, sans épithète supplémentaire, est mentionnée dans une autre inscription à Worms; il s'agit très probablement de la même unité, basée en Germanie Supérieure à Mayence ou à Worms à la période julio-claudienne (Holder 1980, ${ }^{\circ} 493$ ).

- La cohors I Thracum: une cohorte de Thraces (nord de la Grèce), mais dont le surnom de Germanica dans plusieurs diplômes datés de 74, 82, 90 et 116 (diplômes XI, XIV, XXI et $\mathrm{XL}$ ) montre qu'elle a séjourné longtemps en Germanie (avec un recrutement local?). On ne sait si c'est cette cohorte ou une autre du même nom qui participa à la campagne de Caecina contre les Helvètes en 69 . On possède également l'épitaphe d'un préfet de la cohorte à Worms (CIL XIII, $6213=$ = CIRh 897), entre 69 et 100. Ensuite, elle serait partie sur le Danube, lors des campagnes contre les Daces vers 100 ou 101, probablement en compagnie de la legio I Adiutrix (Holder 1980, p. 228 [cohors I Thracum C(ivium) $R$ (omanorum) eq(uitata)]; Cichorius 1893, col. 335-337).

- La cohors VII Breucorum = cohors VII Breucorum c. R. equitata: Cette cohorte des Breuques (Pannonie, Hongrie) est une cohorte montée composée, principalement (car il y a des exceptions) de citoyens romains (c(ivium) $R$ (omanorum). Son stationnement à Worms semble tardif. Des diplômes précisent qu'elle était en Pannonie en 85, puis en Pannonie inférieure en 167. L'inscription mentionnant un préfet de la cohorte à Worms n'est pas datée (CIL XIII, $6213=$ CIRh 897); Holder 1980, p. 225). L'unité est également stationnée

21. À noter que l'ala Indiana Gallorum, l'ala Sebosiana et l'une des alae Hispanorum ont également été stationnées à certaines périodes, totalement ou partiellement, à Mayence - Weisenau (Bernhard 1990, p. 88). à Mayence durant la même période (Holder 1980, n 1241 ; Cichorius 1900, col. 259-260).

- La cohors Raetorum et Vindelicorum : la cohorte des Rhètes et des Vindéliciens (région d'Augsbourg, Bavière) n'est mentionnée qu'au Ir siècle et en Germanie Supérieure par des stèles funéraires de soldats, l'une à Mayence (CIL XIII, 7048) et l'autre à Worms (CIL XIII, 6240, Veiagenus). Il s'agit peut-être de la cohors Raetorum mentionnée par des stèles funéraires de soldats à Mayence $(C I R h 1236)$ et à Worms (CIRh 895), et elle aurait participé à la campagne de Germanicus contre Idistavisus (Tacite, Ann., III, 17 : "Raetorum Vindelicorumque et Gallicae cohortes": Holder 1980, p. 233; également $n^{\circ} 2032$ et 2033 pour sa présence à Mayence; Cichorius 1900, col. 328).

On remarquera la fréquence des mentions d'auxilia à la fois à Mayence et à Worms, ce qui tend à confirmer les hypothèses suivantes :

- la complémentarité évidente de Mayence et de Worms dans le dispositif militaire du Rhin supérieur : Mayence était le siège central d'une puissante garnison légionnaire et le lieu de rattachement des auxilia associés; à Worms et en d'autres points de stationnement sur le Rhin, des forts secondaires étaient défendus par les auxilia;

- les auxilia, ailes et cohortes, étaient soit envoyés par unités entières pour une période donnée à Worms, soit stationnés à Worms sous forme de détachements, ce qui expliquerait les mentions de ces unités dans les deux cantonnements, Worms et Mayence.

\section{Argiotalus et la Gaule de l’ouest}

\section{Argiotalus le Gaulois}

Le monument d'Argiotalus apporte deux noms de Namnètes, ce peuple gaulois établi principalement sur la rive nord de la Loire et de son estuaire. Latinisé, son nom gaulois Argio-talos signifierait "front blanc", "front de neige ", " front brillant " ou "front lumineux " (Fleuriot 1978, p. 25; Delamarre 2003, p. 31, 54 et 288). Il est seul à porter ce cognomen, d'après les indices du CIL et la base de données de l'AE, et on ne connait pas d'autre nom de personne construit sur la même racine. Le nom de son père, Smertulitanos, qui signifierait " qui prévoit large ", "à la large attention » ou « à la vaste sollicitude " (Fleuriot 1978, p. 25; Delamarre 2003, p. 277 et 438) est unique lui aussi, mais sa racine smero- ou smerto-, qui signifierait "prévoyant ", " qui prend soin de ", "pourvoyeur », est associée à l'onomastique divine de Smertrius/Smertrios ou Smertulus, l'Hercule celtique du pilier des Nautes de Paris (CIL XIII, 3026; Esp. 3133; Hatt 2005, p. 35 et 60), naturellement utilisée 
pour de nombreux noms de personne (Smertae, Smertatius, Smertomara, Smertorix, Smertullus, Smertus : Delamarre 2003, p. 277); Smertuccus, chez les Frisons, à Vechten (près d'Utrecht, Pays-Bas : CIL XIII, 8822); Sme(rtullus?) chez les Mandubiens d'Alésia (CIL XIII, 2876); Smertucus (estampille SMERTV du potier Smertucus sur céramique sigillée moulée, à Reims : CIL XIII, 10 010, 1823; Keune 1927, col. 713); Smerca chez les Éduens d'Autun (CIL XIII, 2741) et Smerius à Vienne (Rémy 2005, 665b). On note également à Mayence une Iulia Smertuca (CIL XIII, 8822).

Que savons-nous d'Argiotalus, fils de Smertulitanus, ce Gaulois originaire de la région nantaise? Sa stèle nous apprend qu'il s'est enrôlé (ou a été recruté) dans un corps d'auxiliaires étrangers de l'armée romaine et comme simple cavalier (eques) car, pérégrin, il ne possède qu'un nom unique.

\section{Argiotalus le Namnète}

La stèle de Worms est l'un des cinq documents connus relatifs aux Namnètes ${ }^{22}$, un peuple de la Gaule chevelue dont Auguste dut, vers 27-13 av. J.-C. confirmer le territoire dans le cadre administratif de la civitas Namnetum, la cité des Namnètes, appartenant à la province de Lyonnaise (Deschamps 1992, p. 112-113; Santrot 2008, p. 63-69) ${ }^{23}$.

L'épitaphe d'Argiotalus est la plus ancienne preuve archéologique de l'existence des Namnètes. À la $3^{\mathrm{e}}$ ligne, la graphie Namnis pour Namnetis pourrait être due à la méconnaissance de l'origine du défunt par les héritiers commanditaires du monument ou bien à l'interprétation fautive du lapicide chargé de graver l'épitaphe. Il ne faut pas écarter la possibilité d'un défaut dialectal ou de prononciation : les fautes sont courantes sur les stèles de militaires car le latin des auxiliaires, comme celui des lapicides, est souvent approximatif ou populaire. Étrangers et barbares par nature, au sens premier du terme, comme Argiotalus, les auxiliaires maîtrisaient assez mal le latin, tout comme certains de nos modernes légionnaires leur français d'adoption. Mais une autre hypothèse est plausible : Namnis serait le nom celtique du peuple tandis que Namnetis, l'orthographe correcte attendue $^{24}$, serait l'appellation latinisée de son territoire : «chez

22. Sur les mentions antiques de Nantes et des Namnètes, voir Aubin, 1984, p. 73-76; Provost, 1993, p. 85-86; Santrot, 2008, p. 66-68.

23. Amorcée dès 27 av. J.-C., cette organisation administrative de la Gaule par Auguste a été poursuivie par son gendre, Agrippa, vers 16-13 av. J.-C. (Lepelley, 1998, p. 154).

24. Le dictionnaire Gaffiot indique Namnetes, um, selon César ( $B G$, III, 9, 18) et Pline (NH, IV, 107). La Real-Encyclopädie signale l'orthographe Namnis en se référant à l'épitaphe d'Argiotalus (Nicolas Mathieu, université Pierre Mendès France de Grenoble). les Namnètes ${ }^{25}$ ". Assez puissants pour frapper monnaie (Aubin 1987, p. 17-31; Aubin et Barrandon 1994, p. 205227), ce peuple installé sur le cours inférieur de la Loire n'est mentionné qu'une seule fois par César, vers 50 av. J.-C. ( $B G$, III, 9, 9-10, p. 154-155) puis, sous le vocable de Samnites, par Strabon citant Posidonios, un contemporain de César, vers 18 après J.-C. ${ }^{26}$, puis par Pline l'Ancien, vers $70(\mathrm{NH}$, IV, 107). Sur les occurrences littéraires des références aux Namnètes, voir Cancik 2000, Der Neue Pauly, col. 701.

\section{ConClusion}

Premier témoignage épigraphique de la cité des Namnètes, la stèle d'Argiotalus, fils de Smertulitanus, donne le nom des deux plus anciens habitants connus de la région nantaise, à une époque de "romanisation " active des Gaules mais où des révoltes " nationales ", à causes principalement économiques, persistaient. Elle montre comment un Gaulois, dont le peuple avait été soumis par César, a pu intégrer l'armée romaine par le biais des auxilia, en tant que volontaire ou bien comme contingent livré en " tribut " par la cité sujette des Namnètes on ne sait, mais avec possibilité, au terme d'un long et périlleux service militaire, de prétendre à la citoyenneté romaine. Cela répondait aux besoins de l'armée romaine : les cavaliers gaulois et germains lui apportaient un savoir-faire précieux dans les combats... contre les Germains.

\section{Remerciements}

À Martine Le Jeune, Gilles Leroux, Louis Maurin, Nicolas Mathieu, André Rapin, Michel Reddé et Marie-Hélène Santrot.
25. Sur l'étymologie des Namnètes, certains font référence aux langues brittoniques. Le gallois, le breton et le vieux cornique ont le mot nant ou nans, signifiant "vallée, torrent" (Delamarre, 2003, p. 232). D'autres, se référant aux racines namn- et nanto- (le ruisseau, nager), traduiraient plutôt par "ceux du fleuve" (Lambert, 1995, p. 197). Le mot gaulois nant signifierait aussi confluent (cf. Dubuisson-Aubenay, 2006, p. 511, note marginale 1). Enfin, l'appellatif gaulois nemeton désignerait un lieu consacré (Lacroix, 2007, p. 200-202). Des rapprochements sont proposés avec d'autres noms de peuples comme les Nantuates, en Savoie, ou les Némètes, en Germanie, mais avec quelle légitimité?

26. Une erreur de copiste aurait corrompu "Namnètes" en "Samnites " : [Posidonius] affirme quil y a dans l'océan une petite île, quil situe devant l'embouchure de la Loire et pas tout à fait en haute mer, habitée par les femmes des Samnites possédées de Dionysos et vouées à apaiser ce dieu par des rites mystiques et par toutes sortes de cérémonies sacrées" (Strabon, Géogr., IV, 4, 6). 


\section{Bibliographie}

\section{Sources littéraires}

César, BG : Bellum Gallicum, Livres I-IV, texte établi et traduit par Constans, L. A., $4^{\mathrm{e}}$ éd. rev. et corr., Paris, Les BellesLettres (CUF), 1947; Livres V-VIII, texte établi et traduit par Constans, L. A., revu et corrigé par Balland, A., ibid., 1995, $337 \mathrm{p}$.

Flavius Josèphe, BJ : Bellum Judaicum, texte établi et traduit par Pelletier, A. (s.j.), 1900-1932, Paris, Les Belles-Lettres (CUF), 1975, 220 p.

Notitia Dignitatum : organigramme administratif de l'Empire rédigé sous le règne d'Honorius, en 401, puis en 420, édité par Böcking, Ed., Bonn, A. Marcus, 1839-1853, 3 part., LXVI-539 p., 1209 p., 192 p.

Notitia Dignitatum : édité par Seek, O., Franfort sur le Main, Minerva, 1962, xxx-339 p.

Notitia Galliarum : organigramme administratif rédigé entre 386 et 450 , dans Notitia Dignitatum, édité par SEeK O., 1962 (cf. supra).

Pline, NH : Histoire naturelle, 23-79 av. J.-C., texte établi et traduit par Croisille, J.-M, Paris, Les Belles-Lettres (CUF), 1985, 327 p.

Ptolémée, Géographie: D'après MüLler, K, Claudii Ptolemaei Geographia, Paris, 1883-1901.

Strabon, Géographie : T. II, livres III et IV, texte établi et traduit par Lasserre, F., Paris, Les Belles-Lettres (CUF), 1966, X-242 p.

Tacite, Annales : livres I-III, texte établi et traduit par WuILleumier, P., Paris, Les Belles-Lettres (CUF), 1990, 201 p.

Tacite, Histoires : livres I-III, texte établi et traduit par Goelzer, H., Paris, Les Belles-Lettres (CUF), 1953, xx-212 p.

Table de Peutinger (copie médiévale d'une source datée du règne de Julien - 360-363), copie de Von Scheyb, 1753 redessinée par S. Pineau, O. Pagès et G. Delannay, Paris, éd. Gaule, 1965.

\section{Revues et collections abrégées}

ANRW : Aufstieg und Niedergang der römischen Welt. Geschichte und Kultur Roms im Spiegel der neueren Forschung, TEMPORINI, H.\& HaAse, W. (dir.), Berlin/New York, 1972.

$B A R$ : British archeological reports, Oxford, Hadrian Books.

CIL : Corpus Inscriptionum Latinarum, Berlin, 1885-1965.

CSIR : Corpus Signorum Imperii Romani, Corpus der Skulpturen der römischen Welt, Deutschland, Mayence, Römisch-Germanischen Zentralmuseums, Bonn.

CUF : Collection des Universités de France, Paris, Les Belles Lettres.

Esp. : Espérandieu E., 1908-1922-1981. Recueil général des basreliefs, statues et bustes de la Gaule romaine, 16 vol., Paris, Imprimerie nationale.

RE : Pauly, A., Wissowa, G. et Kroll, W., e.a. (éd.), RealEncyclopädie der klassischen Altertumswissenschaft, Register der Nachträge und Supplemente, Munich, 84 vol. 1893-1983.

\section{Ouvrages et articles}

Alföldy, G., 1968 - Die Hilfstruppen in der römischen Provinz Germania Inferior. Epigraphische Studien, 6, 238 p.

Arbogast, R.-M., Clavel, B., Lepetz, S., Méniel, P. et Yvinec, J.-H., 2002 - Archéologie du cheval, Paris, Errance, 128 p.

Aubin, G., 1984 - L'Antiquité, in AввAD, F. (dir.), La LoireAtlantique des origines à nos jours, Saint-Jean-d'Angély, éd. Bordessoules, p. 63-100.

Aubin, G., 1987 - Répartition des monnaies namnètes. Mélanges offerts au Docteur J.-B. Colbert de Beaulieu, Paris, Le Léopard d'Or, p. 17-31.

Aubin, G. et Barrandon, J.-N, 1994 - Les monnayages armoricains, in Barrandon, J.-N., Aubin, G., Benusiglio, J., Hiernard, J., Nony, D. et Scheers, S., Lor gaulois. Le trésor de Chevanceaux et les monnayages de la façade atlantique, Paris, Cahiers Ernest Babelon 6, p. 141-267 et 373-376 (pl. V-VIII).

Audouin-Rouzeau, F., 1995 - La taille des animaux d'élevage à l'époque romaine et leur exportation. Homme et animal dans l'Antiquité romaine (Actes du colloque de Nantes, 1991). Tours, Centre de recherches A. Piganiol, p. 81-82).

Bernhard, H., 1990 - Die römische Geschichte in RheinlandPfalz, in Cüppers, H. (dir.), Die Römer in Rheinland-Pfalz, Stuttgart, Theiss Verlag, Hambourg, Nikol Verlag, p. 39-168.

Birley, E., 1978 - Alae named after their commanders, Ancient Society, 9, p. 257-273.

Boppert, W., 1992 - Militärische Grabdenkmäler aus Mainz und Umgebung, CSIR Deutschland, II, 5, Mayence/Bonn, 302 p., $120 \mathrm{pl}$.

Boppert, W., 1998 - Römische Steindenkmäler aus Worms und Umgebung, CSIR, Deutschland, II, 10, Germania Superior, Mayence/Bonn, 131 p., 72 pl.

Canciк, H. 2000 - Der Neue Pauly, Encyclopädie der Antike, VIII, Stuttgart, Scheider, col. 701.

Cichorius, C.,1893 - s.v. "Ala ", RE, I, 1, 1893, Stuttgart, col. 1224-1270.

Cichorius, C., 1900 - s.v. " Cohors ", RE, IV, 1, 1900, Stuttgart, col. 231-356.

Cüppers, H. (dir.), 1990 - Die Römer in Rheinland-Pfalz, Stuttgart, Theiss Verlag, Hambourg, Nikol Verlag, 713 p.

Decker, R. V. et Selzer, W., 1976 - s.v. "Mongontiacum ", $A N R W$, II.5.2, col. 465-467.

Delamarre, X., 2003 - Dictionnaire de la langue gauloise. Une approche linguistique du vieux celtique continental, Paris, Errance, $440 \mathrm{p}$.

Desjardins, E., 1876-1893 - Géographie historique et administrative de la Gaule romaine, Paris, Hachette, I (1876), II (1878), III (1885), IV (1893).

Deschamps, S., Guérin, F., Pascal, J. et Pirault, L., 1992 Ratiatum (Rezé, Loire-Atlantique) : origines et développement de l'organisation urbaine, $R A O$, 9, p. 111-127. 
Dixon, K. R. et Southern, P., 1992 - The Roman Cavalry. From the First to the Third Century AD, Londres/New York, B.T. Batsford Ltd, $256 \mathrm{p}$.

Dubuisson-Aubenay (F.-N. Baudot, dit), 1636 - La Bretagne d'après l'Itinéraire de monsieur Dubuisson-Aubenay, 1636, édition commentée par Croix, A. (dir.), Rennes, Presses universitaires de Rennes/Société d'Histoire et d'Archéologie de Bretagne, $1118 \mathrm{p}$.

Durville, G., 1927 - Catalogue du musée archéologique de Nantes ( $2^{\mathrm{e}}$ partie : Musée lapidaire), Nantes, Département de LoireInférieure (Dupas impr.), $146 \mathrm{p}$.

Feugère, M., 1993 - Les armes des Romains, de la République à l'Antiquité tardive, Paris, Errance, 278 p.

Fischer, T., 1999 - Die Römer in Deutschland, Stuttgart, Theiss Verlag, $192 \mathrm{p}$.

Fleuriot, L., 1978 - Quelques remarques sur des noms de Namnètes, Archéologie en Bretagne, 17 ( $\mathrm{n}^{\circ}$ spécial Nantes antique), p. 24-25.

Forest, V., à paraître - Équidés de La Tène finale et de la période romaine en Gaule : approche ostéométrique, in IzAC-IMBERT, L. et BLANCHARD, J.-L. (dir.) - L'exploitation agricole dans son environnement à la fin de l'àge du Fer. Nouvelles approches méthodologiques (Actes de la Table-ronde de l'EHESS de Toulouse, 2004), Toulouse, Archives d'Ecologie préhistorique.

Forest, V. et RodeT-BELARBI, I., 2002 - À propos de la corpulence des bovins en France durant les périodes historiques, Gallia, 59, p. 273-306.

Franke, A., 1936 - «Legio XX Valeria Victrix », RE, XVII.1, col. 1131-1134.

Franke, T., 2000 - "Legio XIV Gemina », in Le Bohec (dir.), 2000, I, p. 190-202 [cf. infra].

GabelmanN, H., 1972 - Die Typen der römischen Grabsteine am Rhein, Bonner Jahrbücher, 72, p. 65-140.

Galliou, P., 1983 et $\mathbf{2 0 0 5}$ - L'Armorique romaine, Brasparts, Les Bibliophiles de Bretagne, $310+30$ p. et rééd. revue et augmentée, Brest, Armeline, $415+$ viII $\mathrm{p}$.

GRÜNEWALD, M., 1990a - Der Römische Nordfriedhof in Worms. Funde von der Mainzer Straße, Worms, Bücher Bessler, $287 \mathrm{p}$.

Grünewald, M., 1990b - Worms, in Cüppers, H. (dir.), 1990, p. 673-679 [cf. supra].

Grünewald, M. et Hahn, E., 2008 - Zwischen Varusschacht und Völkerwanderung. Die römerzeitlichen Gräberfunde aus Worms und Rheinhessen im Museum der Stadt Worms im Andreasstift, Worms, Kunsteverlag Josef Fink, 2 vol., 744 p.

Hamdoune, C., 2007 - Les armes du cavalier africain : de la réalité à la symbolique, in Sauzereau, P. et Van Compernolle, T. (dir.), Les armes dans l'Antiquité, de la technique à l'imaginaire (Actes du colloque international du SEMA, Montpellier, 2003), Montpellier, CERCAM, Université Paul-Valéry - Montpellier 3, Presses universitaires de la Méditerranée, p. 191-211.
Hassal, M., 2000 - The location of legionary fortresses as a response to changes in military strategy: The case of Roman Britain AD 43-84, in Le BoHec (dir.), 2000, II, p. 441-457 [cf. infra].

Hatт, J.-J., 1970 - Les croyances funéraires des Gallo-Romains d'après la figuration des tombes, Revue archéologique de l'Est, 21, (79-80, 1-2), p. 7-97.

Hatт, J.-J., 1986 - La tombe gallo-romaine, suivi de Les croyances funéraires des gallo-romains d'après la décoration des tombes, Paris, Picard, 419 p.

Hatт, J.-J., 2005 - Mythes et dieux de la Gaule, II, diffusion posthume en ligne [http://jeanjacqueshatt.free.fr].

Holder, P. A., 1980 - Studies in the auxilia of the roman army from Augustus to Trajan, Oxford, Hadrian Books (British Archaeological Reports, International Series 70), 352 p.

Holder, P. A., 1982 - The Roman Army in Britain, Londres, Palgrave Macmillan, 173 p.

IHM, 1897 - s.v. « Borbetomagus ", RE, III, 1, Stuttgart, col. 719720.

Junkelmann, M., 1991 - Die Reiter Roms, Der militärische Einsatz, 3, vol. II, Mayence, Philipp von Zabern, 223 p.

Keune, J. B., 1927 - s.v. "Smertucus », RE, III, A, 1, col. 713.

KLeIN, M., 2003 - Römische Helme aus dem Rhein bei Mainz, in Klein, M. (dir.), 2003 - Die Römer und ihr Erbe, Fortschritt durch Innovation und Integration, Mayence, Philipp von Zabern, p. 29-42.

LaCroIx, J., 2007 - Les noms d'origine gauloise, III, La Gaule des dieux, Paris, Errance, 2007, 286 p.

LE Bohec, Y., 1989 et 1990 - L'armée romaine sous le HautEmpire, Paris, Picard, ( $1^{\text {re }}$ et $2^{\mathrm{e}}$ éd.), $287+\mathrm{xxIX}$ p.

Le BoHec, Y. (dir.), 2000 - Les légions de Rome sous le Haut-Empire (Actes du Congrès de Lyon, 1998), Lyon, Centre d'études romaines et gallo-romaines, 3 vol., $756+195$ p.

LEPELleY, C. (dir.), 1998 - Rome et l'intégration de l'Empire (44 av. J.-C.-260 apr. J.-C.), II, Approches régionales du Haut-Empire romain, Paris, PUF, $534 \mathrm{p}$.

Mathieu, F., 2007 - Le guerrier gaulois, du Hallstatt à la conquête romaine, Paris, Errance, 142 p.

Provost, M., 1993 - Le Val de Loire dans l'Antiquité, Paris, CNRS éditions (52 ${ }^{\mathrm{e}}$ suppl. à Gallia), XVI $+425 \mathrm{p}$.

Reddé, M., Brulet, R., Fellmann, R., HaAlebos, J.-K. et Von SCHnurbein, S., 2006 - Les fortifications militaires, in AuperT P. (dir.), L'Architecture de la Gaule romaine, Paris, Maison des Sciences de l'Homme/Bordeaux, Ausonius (coll. Documents d'Archéologie française, 100), $477 \mathrm{p}$.

RÉmy, B. (dir.), 2005 - Inscriptions latines de Narbonnaise, V.3, Vienne, Paris, CNRS éditions (XLIV suppl. à Gallia), 665 p.).

Ritterling, E.,1924 - s.v. " Legio ", RE, XXIII, col. 1186-1328.

Ritterling 1925 - s.v. "Legio ", RE, XII, 2, col. 1761-1765 et XXIV, col. 1328-1829. 
Robinson, H. R., 1975 - The Armour of Imperial Rome, Londres, Arms and Armour Press, 200 p.

Saddington, D. B., 1970 - The Roman Auxilia in Tacitus, Josephus and other early Imperial Writers, Acta Classica, 13, 1970, p. 89-124.

SAddington, D. B., 1975 - The Development of the Roman Auxiliary Forces from Augustus to Trajan, $A N R W$, II.3, 1975, p. 112-137.

Santrot, M.-H. et J., 2003a - Quatre dieux des Santons, perdus et retrouvés, in Bost, J.-P., Roddaz, J.-M. et TAssaux, F., Itinéraire de Saintes à Dougga (Mélanges offerts à Louis Maurin), Bordeaux, Ausonius, (Mémoires, 9, suppl. à Aquitania), p. 71-83.

Santrot, J., 2003b - Le Paon et la divinité " astrale " des thermes, in Bouet, A. (dir.), Thermae Gallicae, Les thermes de Barzan (Charente-Maritime) et les thermes des provinces gauloises, Bordeaux, Ausonius, (Mémoires, 10, suppl. 11 à Aquitania), p. 207-214.

SANTrot, J., 2007 - Lares et laraires en Gaule romaine. Chapelles luxueuses et oratoires populaires, in Baratte, F., Joly, M. et BÉAL, J.-C. (dir.), Luxe et quotidien en Gaule romaine (Actes du colloque de Mâcon, 2005), Mâcon, Musées de Mâcon et Institut de recherche du Val de Saône-Mâconnais, p. 75-104.

Santrot, J., 2008 - Au temps d'Argiotalus, Nantes, Rezé et le port des Namnètes, Annales de Bretagne et des Pays de l'Ouest, 115,1, p. 55-97.
Selzer, W., 1988 - Römische Steindenkmäler. Mainz in römischer Zeit, Mayence, Philipp von Zabern, 267 p.

Spaul, J. E. H., 1994 - A revision and updating of the article written by Conrad Cichorius, $A l a^{2}$. The auxiliary cavalry Units of the prediocletianic Imperial Roman Army, Andover, Nectoreca Press, $327 \mathrm{p}$.

Speidel, M. P., 1994 - Riding for Caesar, The Roman Emperors' Horse Guard, Londres, 1994.

Tassaux, F. et D. (et coll.), 1984 - Aulnay-de-Saintonge : un camp augusto-tibérien en Aquitaine, Aquitania, 2, p. 105-157.

Tassaux, D. et F., 1996 - Les soldats gaulois dans l'armée romaine, in Reddé M. (dir.), L'armée romaine en Gaule, Paris, Errance, p. 147-163.

Tronche, P., 2006 - Aulnay / Rocherou, Aunedonnacum, Charente-Maritime, France, in Reddé, M. et al. (dir.), 2006, p. 205-209 [cf. supra].

Vigneron, P., 1968 - Le cheval dans l'Antiquité gréco-romaine (des guerres médiques aux grandes invasions), contribution à l'histoire des techniques, Nancy, Annales de l'Est (mémoire n 35), 2 vol., $338+105$ p.

Zieling, N., 1989 - Studien zu germanischen Schilden der Spätlatène- und der römischen Kaiserzeit im freien Germanien. Oxford, Hadrian Books, (BAR, International Series 505), 3 vol. 